\title{
An interesting candidate for isolated massive-star formation in the Small Magellanic Cloud ${ }^{\star}$
}

\author{
R. Selier ${ }^{1}$, M. Heydari-Malayeri ${ }^{1}$, and D. A. Gouliermis ${ }^{2}$ \\ ${ }^{1}$ Laboratoire d'Études du Rayonnement et de la Matière en Astrophysique (LERMA), Observatoire de Paris, CNRS, \\ 61 Avenue de l'Observatoire, 75014 Paris, France \\ e-mail: Romain.Selier@obspm.fr \\ 2 Max-Planck-Institut für Astronomie, Königstuhl 17, 69117 Heidelberg, Germany
}

Received 8 November 2010 / Accepted 3 February 2011

\begin{abstract}
Context. The region of the Small Magellanic Cloud (SMC) with which this paper is concerned contains the highest concentration of IRAS/Spitzer sources, $\mathrm{H}_{\mathrm{I}}$ emission, and molecular clouds in this neighboring galaxy. However, it has been the target of very few studies, despite this evidence of star formation.

Aims. We present the first detailed study of the compact H in region N33 in the SMC by placing it in a wider context of massive star formation. Moreover, we show that N33 is a particularly interesting candidate for isolated massive star formation.

Methods. This analysis is based mainly on optical ESO NTT observations, both imaging and spectroscopy, coupled with other archive data, notably Spitzer images (IRAC 3.6, 4.5, 5.8, and $8.0 \mu \mathrm{m}$ ) and 2MASS observations.

Results. We derive a number of physical characteristics of the compact $\mathrm{H}$ II region $\mathrm{N} 33$ for the first time. This gas and dust formation of 7". 4 ( $2.2 \mathrm{pc})$ in diameter is powered by a massive star of spectral type O6.5-O7 V. The compact $\mathrm{H}$ in region belongs to a rare class of $\mathrm{H}_{\text {II }}$ regions in the Magellanic Clouds, called high-excitation blobs (HEBs). We show that this $\mathrm{H}_{\text {II }}$ region is not related to any star cluster. Specifically, we do not find any traces of clustering around N33 on scales larger than $10^{\prime \prime}(\sim 3 \mathrm{pc})$. On smaller scales, there is a marginal stellar concentration, the low density of which, below the $3 \sigma$ level, does not classify it as a real cluster. We also verify that $\mathrm{N} 33$ is not a member of any large stellar association. Under these circumstances, N33 is also therefore attractive because it represents a remarkable case of isolated massive-star formation in the SMC. Various aspects of the relevance of N33 to the topic of massive-star formation in isolation are discussed.
\end{abstract}

Key words. H II regions - stars: early-type - stars: formation - stars: fundamental parameters - ISM: individual objects: N33 Magellanic Clouds

\section{Introduction}

The Small Magellanic Cloud (SMC) provides a unique opportunity for studying star formation in low-metallicity environments. Although a neighboring galaxy, the SMC is considered to be reminiscent of high-redshift galaxies. It has therefore been the subject of numerous surveys and studies at various wavelengths. However, as far as massive star formation is concerned, detailed study of its individual star-forming regions has focused on a relatively small number of cases. Apart from the pre-eminent region N66, to which a considerable amount of research work has been devoted (Heydari-Malayeri \& Selier 2010, and references therein), only several star-forming regions associated with the Shapley's wing (Shapley 1940), such as N81 (Heydari-Malayeri et al. 1999a), N88A (Heydari-Malayeri et al. 1999b), N83/N84 (Lee et al. 2009), and N90 (Cignoni et al. 2009) have been studied in more detail.

In particular, the star-forming regions populating the lower portion of the SMC bar, situated south-west of N66, have so far received little attention. The southern end of the SMC has the particularity of containing the highest concentration of molecular clouds, IRAS/Spitzer sources, and $\mathrm{H}_{\mathrm{I}}$ emission in this neighboring galaxy (Fukui \& Kawamura 2010; Mizuno et al. 2001;

* Based on observations obtained at the European Southern Observatory, La Silla, Chile, Program 69.C-0286(A) and 69.C-0286(B).
Stanimirović et al. 2000, 1999; Blitz et al. 2007; Bolatto et al. 2007; Leroy et al. 2007; Bot et al. 2010). However, higher spatial resolutions and more extended mappings, especially in $\mathrm{CO}$ observations, are required to precisely associate the molecular clouds of that part with individual $\mathrm{H}_{\text {II }}$ regions and study the interaction with massive stars. The $\mathrm{H}_{\text {II }}$ region LHA 115-N33, or in short N33 (Henize 1956), with J2000.0 equatorial coordinates $\alpha=00^{\mathrm{h}} 49^{\mathrm{m}} 29^{\mathrm{s}}$ and $\delta=-73^{\circ} 26^{\prime} 34^{\prime \prime}$, belongs to this part of the SMC. Very few studies have been devoted to it, despite its several interesting characteristics, which the present paper aims to highlight. In particular, as the present paper will show, this object belongs to a small class of Magellanic compact $\mathrm{H}$ II regions, called high-excitation blobs (HEBs; for a review see Heydari-Malayeri et al. 2010). N33 appears also under number 138 in the catalog of emission-line stars and planetary nebulae compiled by Lindsay (1961). In contrast, it is not identified in the optical survey of Davies et al. (1976). Likewise, N33 bears number 297 in the catalog of Meyssonnier \& Azzopardi (1993) among the list of proven or probable SMC compact or small $\mathrm{H}_{\text {II }}$ regions. N33 was detected as the IRAS source 00477-7343 (Helou \& Walker 1988), and in far-IR as the source \#28 in the ISO $170 \mu \mathrm{m}$ catalog (Wilke et al. 2003). N33 was also included in several radio continuum surveys, including the Australia Telescope Compact Array (ATCA) observations at 3 and $6 \mathrm{~cm}$ (Filipović et al. 1998; Indebetouw et al. 2004). 
This region of the SMC also hosts a planetary nebula. This was first suspected by Henize \& Westerlund (1963), who nevertheless could not confirm its presence. Subsequently, Jacoby \& De Marco (2002) detected a planetary nebula candidate at the position (J2000.0) $\alpha=00^{\mathrm{h}} 49^{\mathrm{m}} 35.0^{\mathrm{s}}$ and $\delta=-73^{\circ} 26^{\prime} 36^{\prime \prime} \cdot 9$ (their object number 7 ). This means that the candidate planetary nebula should lie 27" east of the H II region. The reported diameter of the planetary nebula is 7" (Jacoby \& De Marco 2002). Although this planetary nebula is probably unrelated to the $\mathrm{H}$ II region N33, it has created some confusion. In the CDS Simbad database, N33 appears under the heading of planetary nebula. It is also notable that among the 11 references given (covering the 1956-2008 period), only two are directly concerned with the planetary nebula candidate.

As a notable point, this object does not belong to any known stellar association of the SMC (Hodge 1985). In the context of isolated massive star formation studies, this makes N33 particularly interesting. We attempt to show that N33 is an isolated $\mathrm{H}_{\text {II }}$ region powered by a massive star, which has probably formed in isolation. By isolation, we mean not being traceable to an origin in any $\mathrm{OB}$ association. Observational findings suggest that massive stars generally form in groups (e.g., Zinnecker \& Yorke 2007; Schilbach \& Röser 2008; Gvaramadze \& Bomans 2008). There is, however, a statistically small percentage of massive stars $(\sim 5 \%)$ that form in isolation (de Wit et al. 2005; Parker $\&$ Goodwin 2007). It is not yet understood which physical conditions favor the formation of isolated massive stars and how they form. It is pertinent to know whether the same continuous process governs the formation of clusters of massive stars in large molecular clouds and single stars in smaller molecular clouds (see, e.g., Oey et al. 2004, and below Sect. 4). Should the answer be negative, it would be necessary to know the mechanisms responsible for the formation of massive stars in isolation. We discuss several aspects of this topic. The study of this family of massive stars would provide new insights into a clearer understanding of massive stars in general, which is not entirely clear. Apart from studying global aspects of isolated massive star formation, it is necessary to examine each individual case in detail; and N33 provides a rare opportunity for this research work.

This paper is organized as follows. Section 2 presents the observations, data reduction, and the archive data used (Spitzer data, 2MASS data). Section 3 describes our results (overall view, physical parameters, extinction, cluster search, stellar content and the field population, N33 as a HEB, and Spitzer SED fitting). Section 4 presents our discussion, and finally our conclusions are summarized in Sect. 5.

\section{Observations and data reduction}

\subsection{NTT Imaging}

N33 was observed on 26 and 27 September 2002 using the ESO New Technology Telescope (NTT) equipped with the active optics and the Superb Seeing Imager (SuSI2, D'Odorico et al. 1998). The detector consisted of two CCD chips, identified as ESO \#45 and \#46. The two resulting frames were automatically combined to produce a single FITS file, whereas the space between the two chips was "filled" with some overscan columns so that the respective geometry of the two chips was approximately preserved. The gap between the chips corresponds to $\sim 100$ true CCD pixels, or $\sim 8^{\prime \prime}$. The file format was $4288 \times 4096$ pixels, and the measured pixel size $0{ }^{\prime} 085$ on the sky. Each chip of the mosaic covered a field of $5.5 \times 2$. 7 . We refer to the ESO manual $\mathrm{SuSI} 2$ for more technical information.
Nebular imaging was carried out using the narrow-band filters centered on the emission lines $\mathrm{H} \alpha$ (ESO \#884), $\mathrm{H} \beta$ (\#881), and [O III] (\#882) with basic exposures of $300 \mathrm{sec}$ (two exposures each on 26 September, six exposures for $\mathrm{H} \alpha$, and four exposures for $\mathrm{H} \beta$ and [O III] respectively on 27 September). The image quality was quite good during the night, being represented by a seeing of $0 .{ }^{\prime} 6$. We constructed the line-ratio maps $\mathrm{H} \alpha / \mathrm{H} \beta$ and $[\mathrm{O} \mathrm{III}] / \mathrm{H} \beta$ from nebular imaging. We also took exposures using filters ESO \#811 $(B), \# 812(V)$, and \#813 $(R)$ with unit exposure times of $15 \mathrm{~s}$ for $B$ and $V$ and $10 \mathrm{~s}$ for $R$, respectively. The exposures for each filter were repeated twice using ditherings of $5^{\prime \prime}-10^{\prime \prime}$ for bad pixel rejection.

PSF-fitting photometry was obtained for all filters using the DAOPHOT package under IRAF ${ }^{1}$. The magnitudes were then calibrated using the photometric calibration package photcal. To perform this calibration, seven standard stars, belonging to two Landolt photometric groups SA 92 and T Phe (Landolt 1992) were observed at four different airmasses. This led to the determination of the photometry coefficients and zero-points. Those coefficients are in good agreement with the indicative values displayed on the SuSI2 web page.

The aperture corrections were calculated as follows. Starting from one of the frames, we subtracted all stars except the ones used for determining the PSF with the daophot.substar procedure, using our preliminary DAOPHOT photometry and the corresponding PSF. This leads to a frame with only a few bright, isolated stars plus residues from the subtraction. We then performed both aperture and PSF-fitting photometry on those stars, using the same aperture we used for standard stars. The comparison led to aperture corrections of $0.02,0.04$, and $0.03 \mathrm{mag}$ in $B, V$, and $R$, respectively.

During the photometry process, some slight discrepancies between the intensity of the frames were found: this effect was considered to be the consequence of episodic variations in the sky transparency by $7 \%$ at most. In order not to introduce a systematic underestimation of star magnitudes when averaging the frames, we decided to perform photometry on each individual frame.

By cross-correlating the positions of the sources in the various photometry files, we obtained the mean magnitude (average of the 2 mag of each filter) and a decent estimator of the uncertainty in this magnitude (difference between maximum and minimum magnitudes). Finally, the process yielded the photometry of 2400 stars in all three filters. The results for the brightest stars towards N33 are presented in Table 1. The whole photometry is available in electronic form.

\subsection{NTT spectroscopy}

The EMMI spectrograph (Dekker et al. 1986) attached to the ESO NTT telescope was used on 29 September 2002 to obtain several long-slit stellar spectra. The grating was \# 12 centered on $4350 \AA$ (BLMRD mode) and the detector was a Tektronix CCD TK1034 with $1024^{2}$ pixels of size $24 \mu \mathrm{m}$. The covered wavelength range was $3810-4740 \AA$ and the dispersion $38 \AA \mathrm{mm}^{-1}$, giving FWHM resolutions of $2.70 \pm 0.10$ pixels or $2.48 \pm 0.13 \AA$ for a 1.'0 slit. At each position, we took three $10 \mathrm{~min}$ exposure. The instrument response was derived from observations of the calibration stars LTT 7379, LTT 6248, and LTT 7987. The seeing condition was 0.' 8 (FWHM). The identifications of the stars along the slits (see Fig. 2 for the orientations) were based on monitor sketches drawn during the observations.

\footnotetext{
${ }^{1}$ http://iraf.noao.fr
} 
Table 1. Positions and photometry of the main stars in the field of SMC N33 ${ }^{\dagger}$.

\begin{tabular}{lccccccccc}
\hline \hline Star ID & $\alpha(2000)$ & $\delta(J 2000)$ & $V$ & $B-V$ & $V-R$ & $J$ & $H$ & $K$ & Spectral type \\
\hline N33-1 & $00: 49: 29.25$ & $-73: 26: 33.99$ & 15.92 & 0.36 & 0.18 & 13.94 & 13.31 & 12.96 & O6.5-O7 V \\
N33-2 & $00: 49: 29.62$ & $-73: 26: 35.70$ & 16.39 & 1.07 & 0.61 & & & & \\
N33-3 & $00: 49: 28.84$ & $-73: 26: 35.28$ & 17.50 & 0.28 & 0.10 & & & & \\
N33-4 & $00: 49: 24.60$ & $-73: 26: 45.51$ & 11.65 & 0.78 & 0.39 & 10.31 & 9.96 & 9.89 & G5 V (Galactic) \\
N33-5 & $00: 49: 41.75$ & $-73: 26: 41.83$ & 13.04 & 0.77 & 0.40 & 11.72 & 11.38 & 11.33 & \\
N33-6 & $00: 49: 30.58$ & $-73: 26: 50.87$ & 13.56 & 1.76 & 0.95 & 10.48 & 9.62 & 9.39 & K3 I \\
N33-7 & $00: 49: 36.86$ & $-73: 27: 54.49$ & 13.89 & 0.59 & 0.36 & 12.55 & 12.19 & 12.09 & \\
N33-8 & $00: 49: 14.22$ & $-73: 26: 15.80$ & 14.71 & 1.48 & 0.33 & 12.10 & 11.33 & 11.18 & \\
N33-9 & $00: 49: 23.10$ & $-73: 25: 53.87$ & 15.01 & 0.36 & 0.20 & 14.13 & 13.86 & 13.82 & \\
N33-10 & $00: 49: 43.17$ & $-73: 26: 37.02$ & 15.03 & 0.18 & 0.11 & 14.47 & 14.30 & 14.28 & \\
N33-11 & $00: 49: 18.73$ & $-73: 27: 31.13$ & 15.23 & 1.27 & 0.66 & 12.96 & 12.32 & 12.21 & \\
N33-12 & $00: 49: 39.42$ & $-73: 27: 17.54$ & 15.27 & -0.19 & -0.13 & 15.75 & 15.66 & 15.45 & \\
N33-13 & $00: 49: 39.61$ & $-73: 26: 13.45$ & 16.10 & 1.37 & 0.72 & 13.67 & 12.93 & 12.83 & \\
\hline
\end{tabular}

Notes. ${ }^{(\dagger)}$ The $B V R$ photometry results from NTT observations while the JHK measures come from the 2MASS catalog. (‡) Also named SMC 013740 .

Furthermore, EMMI was used on 26 September 2002 to obtain nebular spectra with gratings \# 8 (4550-6650 $)$ and \# 13 4200-8000) in the REMD mode and with grating \# 4 (3650-5350 $\AA$ ) in the BLMD mode. In the REMD mode, the detector was CCD \# 63, MIT/LL, $2048 \times 4096$ pixels of $15^{2} \mu \mathrm{m}^{2}$ each. Spectra were obtained with the slit set in east-west and north-south orientations using a basic exposure time of $300 \mathrm{~s} \mathrm{re-}$ peated several times. The seeing conditions varied between 0.7 . Reduction and extraction of spectra were performed using the IRAF software package. Fluxes were derived from the extracted spectra with the IRAF task SPLOT. The line fluxes were measured by fitting Gaussian profiles to the lines as well as by simple pixel integration in some cases. The nebular line intensities were corrected for interstellar reddening using the formulae given by Howarth (1983) for the LMC extinction, which is very similar to that of the SMC in the visible. The intensities of the main nebular lines are presented in Table 3 where $F(\lambda)$ and $I(\lambda)$ represent observed and dereddened line intensities. The uncertainties are indicated by the capital letters: $\mathrm{A}<10 \%, \mathrm{~B}=10-20 \%$, $\mathrm{C}=20-30 \%$, and $\mathrm{D}>30 \%$.

\subsection{Archive Spitzer and $2 M A S S$ data}

The Spitzer archive data used in this paper come from the $\mathrm{S}^{3} \mathrm{MC}$ project. This is a project to map the star-forming body of the SMC with Spitzer in all seven Infrared Array Camera (IRAC) and Multiband Imaging Photometer for Spitzer (MIPS) bands. We used the IRAC data, obtained in 2005 May, to build a composite image of $\mathrm{N} 33$ and also obtain photometry. The typical PSF of the IRAC images in the 3.6, 4.5, 5.8, and $8.0 \mu \mathrm{m}$ bands is $1^{\prime \prime}$.66 to $11^{\prime \prime} 98$ (Bolatto et al. 2007). The derived photometry for $\mathrm{N} 33$ in the $3.6,4.5,5.8$, and $8.0 \mu \mathrm{m}$ bands are 11.64, $11.20,9.61$, and $7.91 \mathrm{mag}$, respectively, using an integration aperture of 3 pixels, or 3.6" in radius (Charmandaris et al. 2008). Measurements with either slightly larger or smaller apertures do not affect the color results. As for the MIPS fluxes (magnitudes) at the 24 and $70 \mu \mathrm{m}$ bands, they are $0.13 \mathrm{Jy}$ (4.37) and $1.28 \mathrm{Jy}(-0.54)$ respectively. To examine a large field around N33 $(\sim 400 \times 400 \mathrm{pc})$, we used the SAGE-SMC observations. SAGE-SMC is a Spitzer Legacy program that has mapped the entire SMC with IRAC and MIPS. The full mosaics are available at the Spitzer Science Center homepage, SAGE-SMC Data Deliveries.
We also used the JHK photometry provided by the 2MASS point source catalog (http://tdc-www.harvard. edu/catalogs/tmpsc.html), as presented in Table 1. Note that the embedded stars in the $\mathrm{H}_{\text {II }}$ region (\#1, \#2, and \#3) are not resolved in 2MASS data so the JHK photometry of N33-1 corresponds to the whole $\mathrm{N} 33$ compact $\mathrm{H}$ in region.

\section{Results}

\subsection{Overall view}

The images taken with the NTT telescope (Sect. 2.1) have a whole area of $\sim 5^{\prime} \times 5^{\prime}$ corresponding to $\sim 90 \mathrm{pc} \times 90 \mathrm{pc}$ for a distance of $\sim 60 \mathrm{kpc}$, or $m-M=18.94$ mag (Laney \& Stobie 1994). They show a starry field marked by very faint diffuse nebulosity running in the area. A close-up is presented in Fig. 1, which displays the compact $\mathrm{H}_{\mathrm{II}}$ region $\mathrm{N} 33$ towards the center of the image. It has a mean angular radius, $\left(\theta_{\alpha} \cdot \theta_{\delta}\right)^{1 / 2}$, of 3 .' 7 corresponding to a radius of $1.1 \mathrm{pc}$. Broad-band images in $B, V$, and $R$ (Fig. 2) show the presence of three stars towards the $\mathrm{H}$ II region (\#1, \#2, and \#3), whose positions and photometry are listed in Table 1 . The angular separations between these stars are 2'.5 (\#1 and \#2), 2.'5 (\#1 and \#3), and 3".5 (\#2 and \#3). We show that the central star \#1 is the exciting source of the $\mathrm{H}$ II region and therefore physically associated with the nebula. As for stars \#2 and \#3, they may be linked to the $\mathrm{H}_{\text {II }}$ region or alternatively just random field stars. We refer to Sect. 4 for a discussion. Table 1 also contains results for the brightest stars of the field close to the H II region. Star \#6 (also known as SMC 013740 and 2MASS J00493037-7326501) is an SMC supergiant K3 I (Levesque et al. 2006, and below Sect. 3.4). As for star \#4, i.e. the brightest in the vicinity of N33, it is a Galactic dwarf G5 V (see Sect. 3.4). The cross indicates the position of the candidate planetary nebula (Jacoby \& De Marco 2002). Although these authors measure a diameter of 7 " for the planetary nebula, i.e. similar to N33's, this object does not appear in our image. The Spitzer image, a composition of $4.5,5.8$, and $8.0 \mu \mathrm{m}$ bands (Fig. 3), shows N33 as a red nebular object with an interesting diffuse arc or plume hovering over its eastern and north-eastern side. The nature of this feature is not yet clear.

An interesting aspect of the $\mathrm{H}_{\mathrm{II}}$ region N33 is its isolated character, which we deal with amply in Sects. 3.4 and 4. As a matter of fact, no star clusters and emission nebulae are visible in the whole $\sim 90 \times 90$ pc field of the NTT images. We note however that at the position of N33, Bica \& Schmitt (1995) 


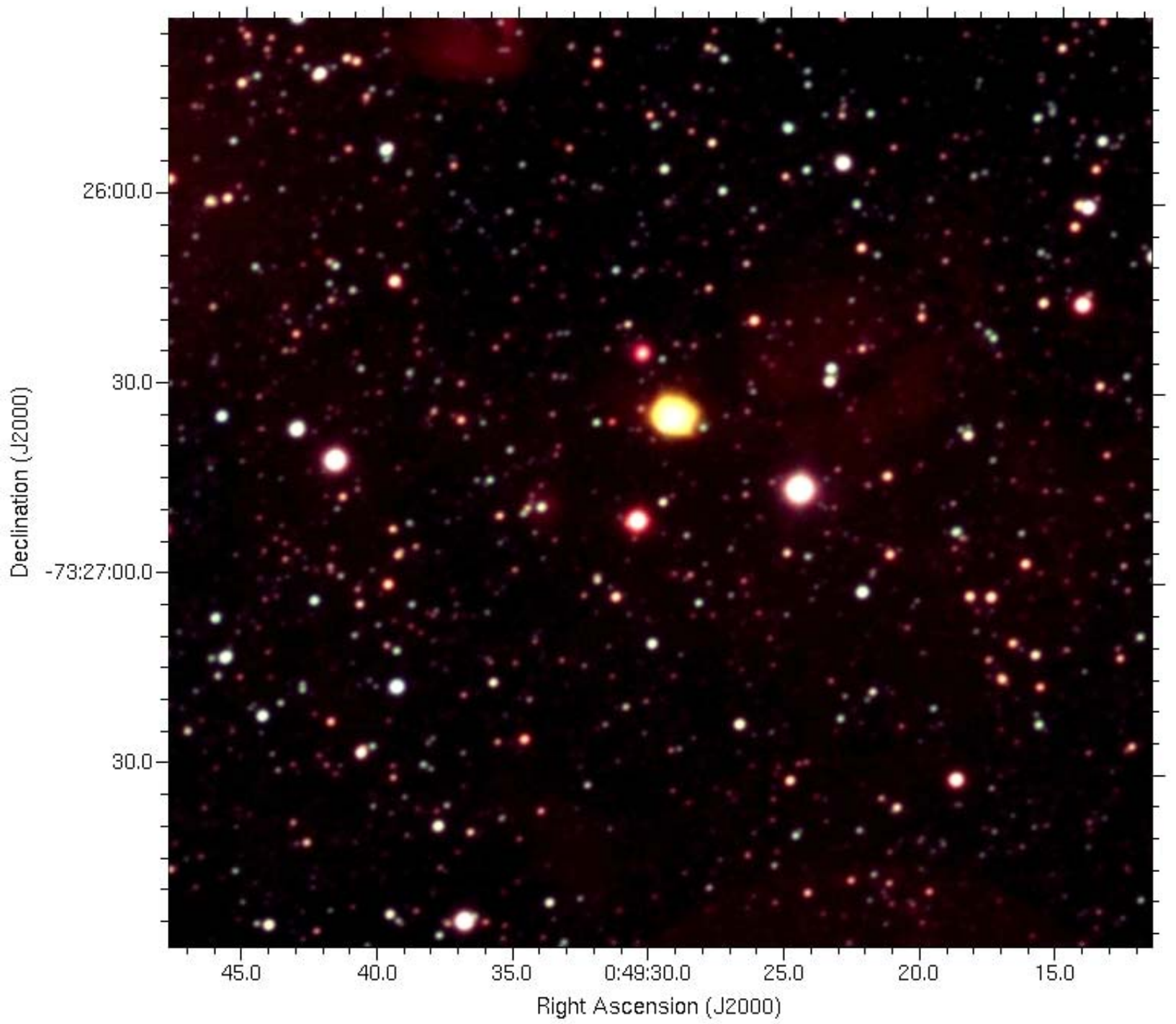

Fig. 1. A composite three-color image of the area of the SMC containing the $\mathrm{H}$ in region N33. The $\mathrm{H}$ in region the bright object is situated above the image center at coordinates $\alpha=00^{\mathrm{h}} 49^{\mathrm{m}} 29^{\mathrm{s}}$ and $\delta=-73^{\circ} 26^{\prime} 34^{\prime \prime}$. See Fig. 2 for the identification of other objects. The image, taken with the ESO NTT/SuSI2, results from the coaddition of narrow-band filters $\mathrm{H} \alpha$ (red), [O III] (green), and $\mathrm{H} \beta$ (blue). Field size $156^{\prime \prime} \times 147^{\prime \prime}(\sim 46 \times 43 \mathrm{pc}$ ). This is a close up of an original image covering a field of $304^{\prime \prime} \times 316^{\prime \prime}$ corresponding to $90 \times 93 \mathrm{pc}$. North is up and east to the left.

identify a compact object, which they classify as an emission nebula, situated in the nebula DEM44 (Davies et al. 1976). Nevertheless, a positional comparison of N33 with DEM44 (located at 0:48:58.53-73:25:39.76, J2000) shows that N33 lies about $130 \mathrm{pc}$ away from the center of the nebula, and about $70 \mathrm{pc}$ from its closest edge, considering its dimensions of $7^{\prime} \times 5^{\prime}$, as provided by Davies et al. (1976). As a consequence, there is no apparent association of N33 with DEM44. We also examined a larger field, $\sim 400 \times 400 \mathrm{pc}$, around N33 using the Spitzer SAGESMC archive data. Similarly, no conspicuous emission source is present out to a projected distance of 200 pc north-west of N33. At that position, an extended emission object shows up, that should correspond to the SMC H II regions N22/N23. Moreover, a minor source is seen south of $\mathrm{N} 22 / \mathrm{N} 23$ at a projected distance of $\sim 160$ pc from N33.

\subsection{Physical parameters}

The total $\mathrm{H} \beta$ flux of the compact $\mathrm{H}$ II region N33 was derived using the following procedure. First we calculated the relative
$\mathrm{H} \beta$ flux in an imaginary $1^{\prime \prime}$ slit passing through the $\mathrm{H} \beta$ image with respect to the total flux emitted by the whole $\mathrm{H}_{\text {II }}$ region. This value was then compared with the absolute flux obtained from the spectra. The total $\mathrm{H} \beta$ flux thus obtained was $F(\mathrm{H} \beta)=$ $1.05 \times 10^{-12} \mathrm{erg} \mathrm{cm}^{-2} \mathrm{~s}^{-1}$. Studies of the extinction in the LMC and the SMC reveal reddening laws that are similar to the average Galactic law for the optical and near-IR regions (Howarth 1983; Prévot et al. 1984; Bouchet et al. 1985). Considering the extinction law for the LMC (Howarth 1983), we computed the reddening corrected intensity $I(\mathrm{H} \beta)=4.91 \times 10^{-12} \mathrm{erg} \mathrm{cm}^{-2} \mathrm{~s}^{-1}$. We derived a luminosity of $2.2 \times 10^{36} \mathrm{erg} \mathrm{s}^{-1}$, or $550 L_{\odot}$, for $\mathrm{N} 33$ at $\mathrm{H}(\beta)$. This luminosity corresponds to a flux of $5.4 \times$ $10^{47} \mathrm{H} \beta$ photons $\mathrm{s}^{-1}$, or a Lyman continuum flux of $4.7 \times$ $10^{48}$ photons s $^{-1}$ for the star, assuming that the $\mathrm{H}$ II region is ionization bounded. The exciting star needed to provide this flux should have an effective temperature of $\sim 36000 \mathrm{~K}$ or be of spectral type about O6.5-O7 V, for Galactic metallicity (Martins et al. 2005). This may however be a lower limit because of photon loss in a density-bounded $\mathrm{H}$ in region.

A number of the derived physical parameters of the compact $\mathrm{H}_{\text {II }}$ region are summarized in Table 2. The mean angular 


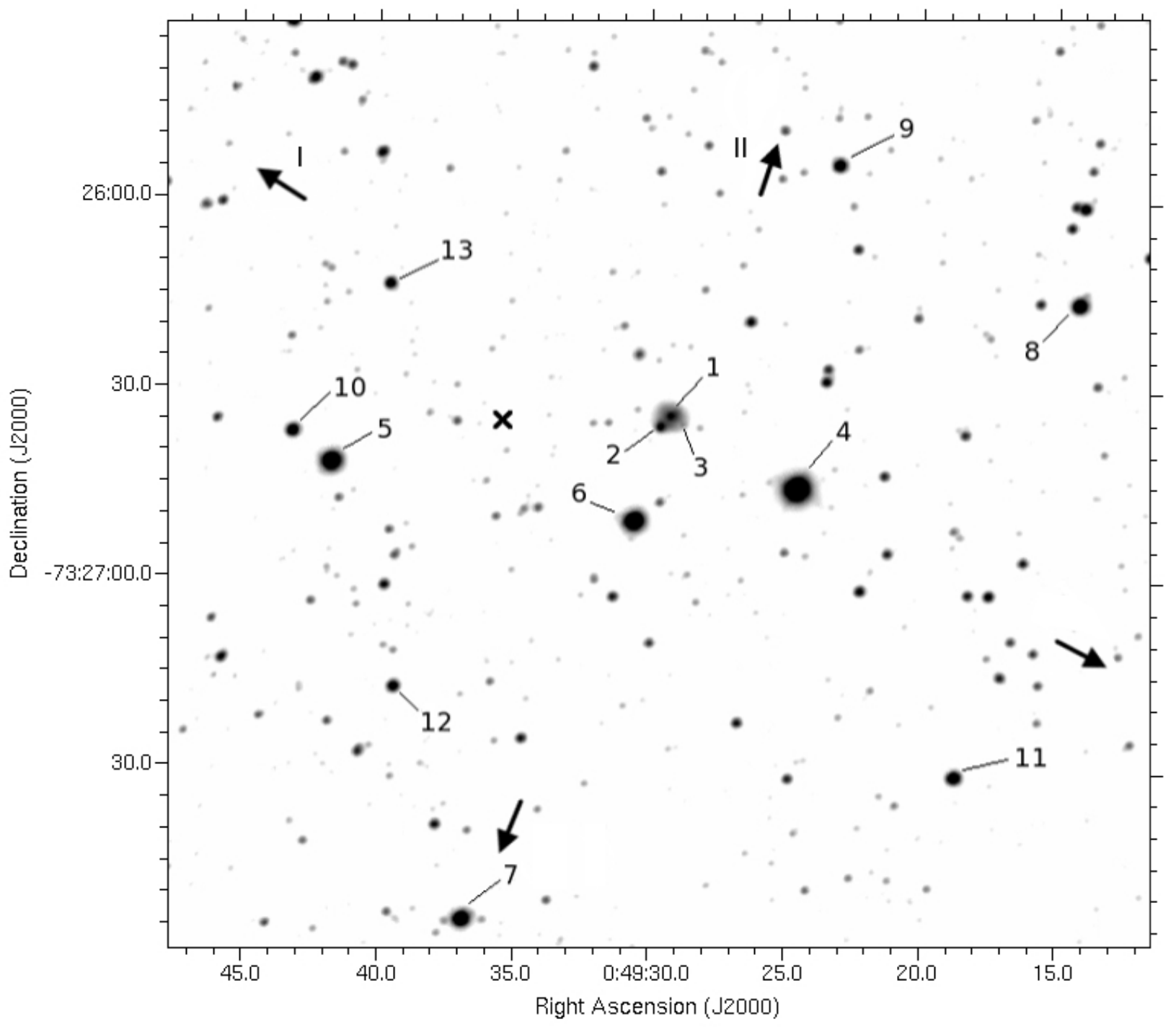

Fig. 2. A broad-band image of the SMC N33 region obtained through filter $R$ (ESO \#813). Same field size and orientation as in Fig. 1. The arrows I and II show the directions of the spectrograph slit. Seeing 0'.6 FWHM. The H II region apparently contains three stars (numbered \#1, \#2, and \#3). The brightest stars of the field are also numbered. The cross indicates the position of the candidate planetary nebula reported by Jacoby \& De Marco (2002).

Table 2. Some physical parameters of the compact $\mathrm{H}_{\text {II }}$ region SMC N33.

\begin{tabular}{lccccccc}
\hline \hline $\begin{array}{l}\theta \\
\left(^{\prime \prime}\right)\end{array}$ & $\begin{array}{c}r \\
(\mathrm{pc})\end{array}$ & $\begin{array}{c}I(\mathrm{H} \beta)^{\dagger} \\
\mathrm{erg} \mathrm{s}^{-1} \mathrm{~cm}^{-2} \\
\times 10^{-12}\end{array}$ & $\begin{array}{c}T e \\
(\mathrm{~K})\end{array}$ & $\begin{array}{c}N e^{\ddagger} \\
\mathrm{cm}^{-3}\end{array}$ & $\begin{array}{c}\left\langle n_{\mathrm{e}}\right\rangle \\
\mathrm{cm}^{-3}\end{array}$ & $\begin{array}{c}M_{\mathrm{gas}} \\
\left(M_{\odot}\right)\end{array}$ & $\begin{array}{c}N_{\mathrm{L}} \\
\mathrm{ph} \mathrm{s}^{-1} \\
\times 10^{48}\end{array}$ \\
\hline 3.7 & 1.1 & 4.91 & 12540 & 450 & 380 & 64 & 4.7 \\
\hline
\end{tabular}

Notes. ${ }^{(\dagger)}$ Corrected for reddening with $c(\mathrm{H} \beta)=0.67 .{ }^{(\ddagger)}$ Estimated from the $\left[\mathrm{S}_{\mathrm{II}}\right]$ ratio.

radius of the $\mathrm{H}$ II region, corresponding to the FWHM of crosscuts through the $\mathrm{H} \alpha$ image, is given in Col. 1. The corresponding physical radius, obtained using a distance modulus of $m-M=18.94$ mag (Laney \& Stobie 1994) is presented in Col. 2. The dereddened $\mathrm{H} \beta$ flux obtained from a reddening coefficient of $c(\mathrm{H} \beta)=0.67$ is given in Col. 3. This reddening coefficient, derived from the mean $\mathrm{H} \alpha / \mathrm{H} \beta$ ratio of 4.6, corresponds to the whole $\mathrm{H}_{\text {II }}$ region. It is different from the value found from the nebular spectrum (Table 3 ) because, in contrast, the spectrum belongs to a particular position and therefore does not cover the whole region. The electron temperature calculated from the forbidden-line ratio [O III] $\lambda \lambda 4363 /(4959+5007)$, with an uncertainty of $4 \%$, is given in Col. 4 . The electron density, estimated from the ratio of the [S $\mathrm{S}_{\text {II }}$ ] doublet $\lambda \lambda 6717 / 6731$, is presented in Col. 5. It is accurate to $\sim 80 \%$. It is well-known that the [S II] lines characterize the low-density peripheral zones of $\mathrm{H}_{\text {II }}$ regions (see below for corroboration). Column 6 gives the rms electron density, $\left\langle n_{\mathrm{e}}\right\rangle$, calculated from the $\mathrm{H} \beta$ flux, the radius, and the electron temperature, $T_{\mathrm{e}}$, assuming that the $\mathrm{H}_{\text {II }}$ region is an ionization-bounded Strömgren sphere. Furthermore, the total mass of the ionized gas, calculated from the $\left\langle n_{\mathrm{e}}\right\rangle$ with the previously noted Strömgren sphere assumption is presented in Col. 7. The ionization is produced by Lyman continuum photon flux given in Col. 8.

\subsection{Extinction}

The average value of the Balmer decrement towards the $\mathrm{H}$ II region N33 is about 4.6 , corresponding to $A_{\mathrm{V}}=1.5 \mathrm{mag}$, using 
Table 3. Nebular line intensities of the SMC compact H in region N33.

\begin{tabular}{|c|c|c|c|c|}
\hline$\lambda(\AA)$ & Iden. & $F(\lambda)$ & $I(\lambda)$ & Accuracy \\
\hline 3727,29 & [O $\mathrm{II}]$ & 77.1 & 108.8 & $\mathrm{~A}$ \\
\hline 3797 & $\mathrm{H} 10$ & 2.4 & 3.3 & B \\
\hline 3835 & H9 & 3.0 & 4.1 & B \\
\hline 3869 & {$[\mathrm{Ne}$ III] } & 15.2 & 20.3 & A \\
\hline 3889,90 & $\mathrm{He}$ I + H8 & 8.6 & 11.4 & B \\
\hline 3968,70 & {$[\mathrm{Ne} \mathrm{III}]+\mathrm{H} \epsilon$} & 11.8 & 15.2 & A \\
\hline 4101 & $\mathrm{H} \delta$ & 14.0 & 17.2 & A \\
\hline 4340 & $\mathrm{H} \gamma$ & 29.1 & 33.3 & A \\
\hline 4363 & [O & 4.8 & 5.5 & B \\
\hline 4471 & $\mathrm{He}_{\mathrm{I}}$ & 3.4 & 3.7 & $\mathrm{C}$ \\
\hline 4861 & $\mathrm{H} \beta$ & 100.0 & 100.0 & A \\
\hline 4959 & [O III] & 148.3 & 145.1 & A \\
\hline 5007 & [O III] & 444.8 & 430.8 & A \\
\hline 5876 & $\mathrm{He}_{\mathrm{I}}$ & 13.8 & 11.5 & B \\
\hline 6300 & {$\left[\mathrm{O}_{\mathrm{I}}\right]$} & 4.4 & 3.4 & $\mathrm{C}$ \\
\hline 6312 & {$[\mathrm{~S}$ III] } & 2.3 & 1.8 & $\mathrm{C}$ \\
\hline 6363 & [O I $]$ & 1.3 & 1.0 & $\mathrm{D}$ \\
\hline 6548 & {$[\mathrm{~N}$ II] } & 4.1 & 3.1 & $\mathrm{C}$ \\
\hline 6563 & $\mathrm{H} \alpha$ & 380.0 & 286.0 & A \\
\hline 6584 & {$\left[\mathrm{~N}_{\text {II }}\right]$} & 10.3 & 7.8 & B \\
\hline 6678 & $\mathrm{He}_{\mathrm{I}}$ & 4.1 & 3.1 & B \\
\hline 6716 & {$[\mathrm{~S}$ II $]$} & 10.1 & 7.5 & B \\
\hline 6731 & {$[\mathrm{~S}$ II $]$} & 9.3 & 6.9 & B \\
\hline 7065 & $\mathrm{He}_{\mathrm{I}}$ & 4.3 & 3.1 & $\mathrm{C}$ \\
\hline 7135 & [Ar III] & 11.9 & 8.4 & B \\
\hline 7236 & [Ar IV $]$ & 1.0 & 0.7 & D \\
\hline 7323 & {$\left[\mathrm{O}_{\mathrm{II}}\right]$} & 10.1 & 7.0 & $\mathrm{C}$ \\
\hline 7751 & [Ar III] & 3.7 & 2.4 & $\mathrm{C}$ \\
\hline
\end{tabular}

the extinction law for the LMC with $R=3.1$ (Howarth 1983). The most extincted part of the $\mathrm{H}$ II region is its northeast border, where the $\mathrm{H} \alpha / \mathrm{H} \beta$ ratio reaches a value of $6.5\left(A_{\mathrm{V}}=2.5 \mathrm{mag}\right)$. Interestingly, the IR elongated structure (Sect. 3.1), which runs from north to south-east (i.e. from the area indicated by the upper circle in Fig. 3), happens to be adjacent to this higher extinction area. The extinction towards star \#1 can be derived from a second method. O-type stars have an intrinsic color of $B-V=-0.28 \mathrm{mag}$ (Martins \& Plez 2006). This yields a color excess of $E(B-V)=0.64 \mathrm{mag}$ or a visual extinction of $A_{\mathrm{V}}=2.0$ mag for star \#1, in good agreement with the result from the Balmer decrement.

Moreover, the extinction towards N33 was estimated by a third method using radio continuum observations. N33 appears as the source B0047-7343 in the Parkes radio continuum survey at 3 and $6 \mathrm{~cm}$, which had beam-sizes of 2.7 and 13'8, respectively (Filipović et al. 1998). Higher resolution observations of this object in the same wavelength range were obtained by Indebetouw et al. (2004), who in their search for ultracompact and buried $\mathrm{H}_{\mathrm{II}}$ regions in the Magellanic Clouds, used the Australia Telescope Compact Array (ATCA) in radio continuum emission at 3 and $6 \mathrm{~cm}$ with synthesized beams of 1".5 and $2^{\prime \prime}$, respectively. Since the beam-widths are $1^{\prime \prime} .5$ and $2^{\prime \prime}$, respectively, the radio continuum observations do not sample the entire $\mathrm{H}_{\text {II }}$ region. To compare the radio continuum fluxes with that of $\mathrm{H} \beta$, we corrected the $\mathrm{H} \beta$ flux. We computed these from the ratio of the measured $\mathrm{H} \beta$ emission flux with different apertures (1.'5 and $\left.2^{\prime \prime}\right)$ to the total N33 flux. The resulting extinction, $A_{\mathrm{V}}=1.9$ mag, although comparable with that obtained using the previously mentioned methods in the optical range, may be an underestimate if the flux is not uniformly distributed over the $\mathrm{H}$ II region and the smaller radio lobes miss a part of it.

\subsection{Cluster search}

We perform a cluster analysis technique on the stellar photometric catalog from our SuSI2 imaging, to identify any potentially important stellar concentration at the surroundings of the compact $\mathrm{H}_{\text {II }}$ region N33 that would suggest membership of its central star to a stellar cluster. Our identification method is based on star counts in quadrilateral grids for the construction of stellar density maps in the area of interest (see e.g., Gouliermis et al. 2000). We performed star counts on our complete photometric catalog of 2400 sources. This was done by counting the stars in grids according to their celestial positions, as they are defined by our astrometry. The method is sensitive to three factors, namely 1) the stellar numbers; 2) the size of each grid element, which defines the smallest possibly detectable cluster (the resolution element in the density maps); and 3) the size of the available field-of-view (FoV). This is due to the isopleths of the stellar density maps whose steps are defined to be equal to the standard deviation, $\sigma$, of the total average stellar density (of stars per grid element) of the considered field. We refer to the latter as the "background density". We consider any stellar concentration revealed by the isopleths, that correspond to stellar density of $3 \sigma$ above the background and higher, as statistically important, and therefore as a candidate true cluster.

The application of star counts to the whole observed FoV allows us to identify any possible membership of N33 to any large stellar concentration of size on the order of $\sim 100 \mathrm{pc}$. It should be noted that the gap between the chips of SuSI2 introduces a lack of stars along it in every photometric catalog derived for each filter. As a consequence, the final combined photometric catalog also contains a lack of stars along a 100 pixel thick line vertically crossing the middle of SuSI 2 frame. While this introduces a problem into the consistency of the detection, the gap is fortunately away from the $\mathrm{H}_{\text {II }}$ region, and hence its effect on the cluster detection at the immediate vicinity of N33 is not important. We constructed the stellar density maps for the SuSI2 FoV using a grid of $25 \times 25$ elements, corresponding to a physical scale of about $3 \mathrm{pc}$ per grid element, which defines the smallest possible stellar concentration that can be revealed in the isodensity maps. These maps are constructed for all the stars, as well as for selected "blue stars" with $B-V \leq 0.55$ mag, mostly representing the main-sequence stellar population and "red stars" selected to have colors $B-V>0.55$ (see color-magnitude diagram of Fig. 7), which represent the evolved stellar population of the general SMC field. In Fig. 4, the stellar isodensity maps at the vicinity of N33, i.e. in the close-up field of Figs. 1 and 2, are shown along with the corresponding stellar map.

As seen from the density maps of Fig. 4, all the isopleths around $\mathrm{N} 33$ appear below the $3 \sigma$ density threshold. This clearly implies that, considering the factors on which the star counts method depends, there is no statistically significant stellar cluster around N33, of size larger than 3 pc. Our search for stellar clustering around N33 is based on the complete stellar catalog constructed from our SuSI2 photometry. This catalog, according to the evolutionary models, covers stars with masses as low as about $2 M_{\odot}$ corresponding to late A-type dwarfs. It should be noted that the low completeness in faint stars may not allow us to have a significant density peak around N33 simply because we do not see these stars. Nevertheless, a small stellar clump of projected size $\simeq 18^{\prime \prime} \times 26^{\prime \prime}(5 \mathrm{pc} \times 7 \mathrm{pc})$ is revealed around $\mathrm{N} 33$ at the $1 \sigma$ density level and the isodensity maps of Fig. 4 suggest that it mainly consists of main-sequence stars. However, its low density, reaching the $3 \sigma$ level only at its very central peak, does not classify it as a real cluster. Moreover, from our 


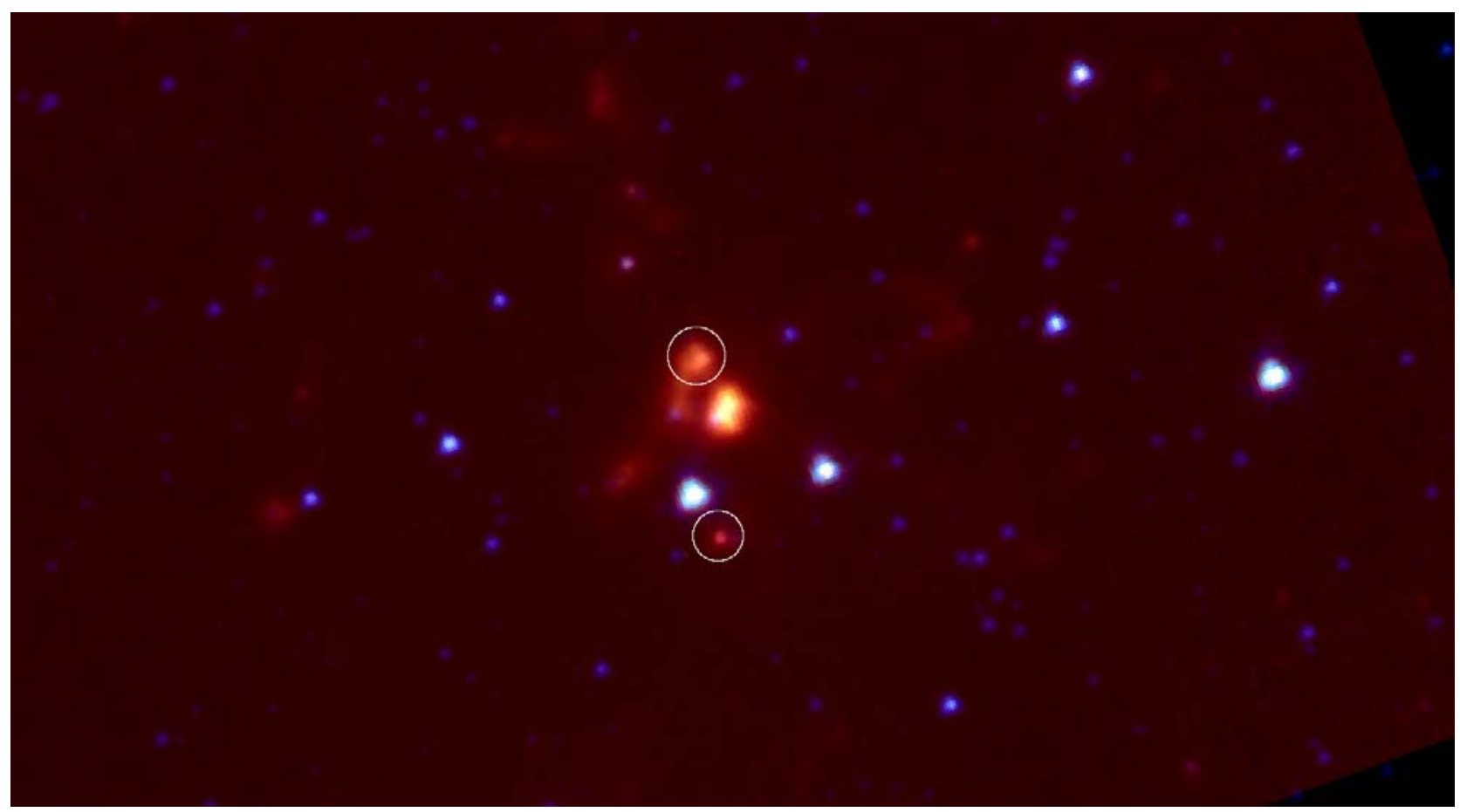

Fig. 3. A composite image of the SMC N33 region obtained with Spitzer IRAC data. The object at the center is the H II region N33. The two other bright sources are stars \#4 and \#6 (see Fig. 2). The $4.5 \mu \mathrm{m}$ band is represented in blue, the $5.8 \mu \mathrm{m}$ band in yellow, and the $8.0 \mu \mathrm{m}$ band in red. The compact $\mathrm{H}$ in region $\mathrm{N} 33$ is the red nebular object at center. Note the diffuse emission curl over the main body of the $\mathrm{H}$ in region running from north (inside the upper circle) to south-east. The circles enclose the young stellar object (YSO) candidates S3MC_J004930.12-732623.42 and S3MC_J004929.07-732658.56 reported by Bolatto et al. (2007). However, the true nature of these objects and their relation with N33 are not clear; see the text (Sect. 4). Field size $280^{\prime \prime} \times 160^{\prime \prime}(\sim 83 \times 47 \mathrm{pc})$. North is up and east to the left.

cluster analysis in the whole observed FoV we assess that N33 does not seem to belong to any larger stellar concentration on a 100 pc length-scale. A possible reason for not identifying any parent stellar structure for N33 is that the H II region may indeed be part of a larger stellar aggregate, the size of which is much larger than our observed FoV. In this case, our search for important density enhancements would take place within the system itself, without apprehending its existence, thus our average background density would not represent the real background, but the high stellar density levels of the large concentration. However, previous searching-by-eye investigations by Hodge (1985) and Bica \& Schmitt (1995), on FoVs larger than ours do not detect any large stellar concentration towards this region. The only indication of a large stellar concentration in this region is found by Battinelli (1991), who identified a candidate stellar association, that seems to coincide with the larger nebula DEM44. This author applied a cluster analysis technique, the path linkage criterion, to previous catalogs of OB-type stars to identify candidate $\mathrm{OB}$ associations in the SMC. However, this method is applied only to the OB stars, and therefore is biased towards large-scale "connections" of these stars without any supporting information about their physical relation or any interaction to each other. Moreover, the identification of large $\mathrm{OB}$ groups based solely on the positions of the stars can be challenged, because it has been confirmed that young stellar systems may lose a significant fraction of their massive stars at the very beginning of their evolution (e.g., Gvaramadze \& Bomans 2008), and therefore OB stars may be mistakenly considered as members of a system, while being runaways from another. Under these circumstances, and because DEM44 does not seem to contain N33, the nebula coinciding with a candidate $\mathrm{OB}$ association cannot be considered proof that N33 does belong to this large stellar concentration.
To assess the clustering behavior of the observed stars in the region of N33, we construct the two-point correlation function (TPCF), which determines the distance between all possible pairs of stars. This method, as applied by e.g. Gomez et al. (1993) to study the distribution of young stars in a Galactic star-forming region, considers the excess number of pairs in the actual distribution over a random distribution. Here, we apply the method as extended by Larson (1995), who introduced the assessment of stellar clustering in terms of the average surface density of companions, $\Sigma_{\mathrm{c}}(\theta)$, as a function of the projected angular separation, $\theta$. We measured $\Sigma_{\mathrm{c}}(\theta)$ and correlated it to the corresponding angular separation for the blue stars in the whole observed FoV, as well as for the stars covered by the closeup field centered on N33. The constructed TPCFs are shown in Fig. 5 drawn with a red line for the close-up field and blue for the whole observed field. In both samples, a clear change in the correlation $\Sigma_{\mathrm{c}}(\theta)$ appears at $\theta \simeq 10^{\prime \prime}$, which at the distance of the SMC corresponds to about 3 pc. We note that this limit is similar to that measured for the small stellar clump seen at the position of N33 in the density maps of Fig. 4. For larger separations $\left(\theta>10^{\prime \prime}\right)$, the relation $\Sigma_{\mathrm{c}}(\theta)$ becomes almost flat, with $\Sigma_{\mathrm{c}} \propto \theta^{-0.2}$. This almost-constant density of companions for larger separations indicates a random distribution of stars and therefore no clustering of stars at scales larger than $3 \mathrm{pc}$. On the other hand, for smaller scales the average density of companions correlates linearly with the separation as $\Sigma_{\mathrm{c}} \propto \theta^{-1.1}$, providing clear evidence of self-similar clustering on scales as small as the smallest resolved separation, and eventually in the binary and multiple systems regime. This implies that stellar clustering in the observed FoV seems to occur only at scales smaller than about $3 \mathrm{pc}$, in agreement with the findings of our cluster analysis above. In conclusion, from our analysis we find that 

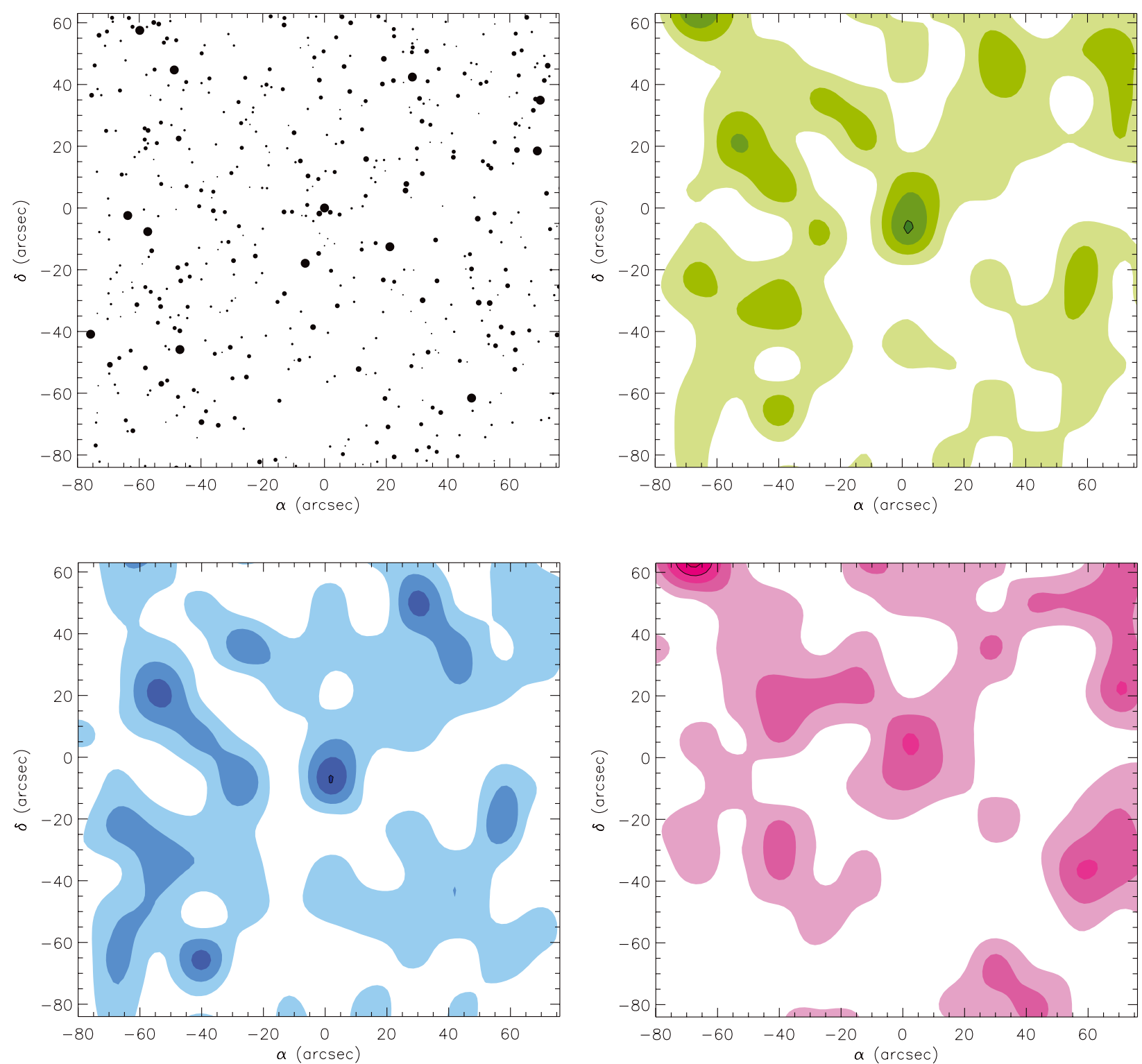

Fig. 4. Isodensity contour maps constructed to search for stellar clustering around the compact SMC H in region N33. In the top-left panel, the stellar map for the close-up field of view centered on N33 (position 0,0). The stellar density maps refer to the same close-up field of N33. They are shown as they are constructed for all observed stars (green map, top-right panel), for blue (main sequence) stars (blue map, bottom-left panel), and red evolved stars (red contour map, bottom-right panel). Isopleths are plotted starting at the average background stellar density and in steps of $\sigma$ above this level. No stellar concentration with density $\geq 3 \sigma$ above the background is identified in the maps. Only the central part of the small stellar clump encircling N33 in the green and blue maps reaches the $3 \sigma$ density level.

the $\mathrm{H}$ II region $\mathrm{N} 33$ does not seem to belong to any large stellar concentration. On smaller scales, we identify a peak in the stellar density around N33, with a stellar mass of roughly $100 M_{\odot}$, which nevertheless is very loose and therefore should be considered as a random density fluctuation rather than a real cluster.

\subsection{Stellar content and the field population}

We obtained two spectra in our program of stellar spectroscopy (Sect. 2.2). However in spite of the relatively good seeing conditions ( 0.7 FWHM), extracting uncontaminated spectra is not straightforward. The compact $\mathrm{H}_{\text {II }}$ region (mean size 7".4) has strong emission lines that fill in the absorption lines of the embedded stars, in particular those of He I. Nevertheless, He II absorption lines at $\lambda \lambda 4200,4541$, and 4686 are certainly present, albeit with weak $\mathrm{S} / \mathrm{N}$ ratio, in the spectra uncorrected for nebular lines (Fig. 6). This agrees with the presence of an $06.5-07 \mathrm{~V}$ spectral type, as inferred from the $\mathrm{H} \beta$ flux measurement (see Sect. 3.2). The estimated spectroscopic mass of an O6.5 V star is $29 M_{\odot}$ and its effective temperature $T_{\text {eff }}=36800 \mathrm{~K}$ (Martins et al. 2005). We also note that Indebetouw et al. (2004) derive a spectral type of $08.5 \mathrm{~V}$ for the exciting source of N33 from their radio continuum observations at 3 and $6 \mathrm{~cm}$. As underlined above, these ATCA observations do not sample the entire $\mathrm{H}$ II region. Furthermore, the presence of the He II absorption lines in the spectrum of the exciting star rules out a confusion of N33 with a planetary nebula.

The spectrum of star \#4, the brightest object close to the $\mathrm{H}$ II region, is presented in Fig. 6. Using classification criteria for late-type stars (Jaschek \& Jaschek 1987; Gray \& Corbally 2009), star \#4 can be assigned the subtype G5. This spectral type is based on the strength of the Ca II $\lambda 4227$ line and the 
R. Selier et al.: SMC compact $\mathrm{H}$ in region N33

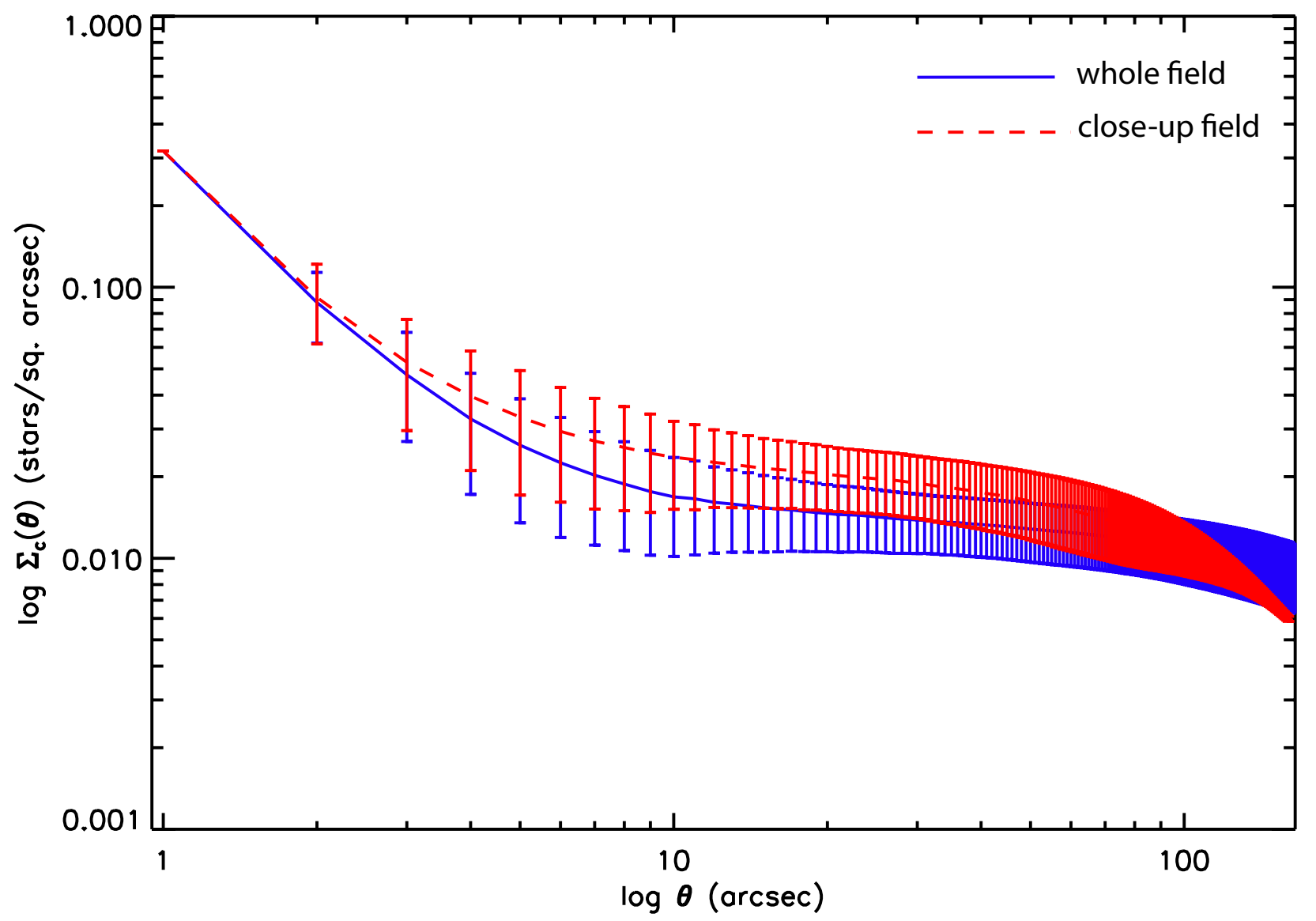

Fig. 5. Average surface density of stellar companions as a function of projected angular separation from each blue star in the whole observed area (blue solid line) and in the region of the vicinity of the H II region N33 (red dashed line). Error bars are indicated. From both curves, one can distinguish a break in the correlation at almost the same separation of $\theta \simeq 10^{\prime \prime}$, i.e., 3 pc. This scale separates any large stellar clustering from the small binary/multiple stellar clumps.

ratios $\mathrm{Fe}_{\mathrm{I}} \lambda 4046 / \mathrm{H} \delta$ and $\mathrm{Fe}$ I $\lambda 4325 / \mathrm{H} \gamma$. This result agrees with the measured colors: $B-V=0.78, V-R=0.39, V-K=1.76$, $H-K=0.07$, and $J-K=0.42$ mag (Koornneef 1983). In terms of luminosity class, it is a dwarf, as suggested by the line ratios $\mathrm{Sr}$ II $\lambda 4077 / \mathrm{Fe}$ I $\lambda 4046$ and Y II $\lambda 4376 / \mathrm{Fe}_{\mathrm{I}} \lambda 4383$. We also note that the $\mathrm{Ca}$ II $\mathrm{K}$ and $\mathrm{H}$ lines do not have extremely broad and damped wings as expected in supergiants. This star should have an absolute magnitude $M_{\mathrm{V}}=+5.1$ (Jaschek \& Jaschek 1987). However, this value is at odds with the observed absolute magnitude of $M_{\mathrm{V}}=-7.31$ assuming that it belongs to the SMC. This discrepancy indicates that star \#4 is a Galactic member. Additional evidence of the Galactic membership of this star is its relatively low radial velocity. In contrast, star \#6, the other bright field star, has a radial velocity of $156.4 \mathrm{~km} \mathrm{~s}^{-1}$ which confirms its SMC membership. Figure 6 also displays the spectrogram of star \#6, which is much redder $(V-K=4.17 \mathrm{mag}$, see also Table 1$)$. We classify this star as an early-K type supergiant, in agreement with its previously reported classification, K3 I (Levesque et al. 2006).

Figure 7 displays the color-magnitude diagram of the population of stars in the entire NTT field of $304^{\prime \prime} \times 316^{\prime \prime}$ $(90 \times 93 \mathrm{pc})$, for a cut-off magnitude of $V=20.5$. We also overplotted four isochrones with ages $18 \mathrm{Myr}, 56 \mathrm{Myr}, 500 \mathrm{Myr}$, and 1 Gyr for metallicity $Z=0.004$ (Lejeune \& Schaerer 2001). A fifth isochrone of $14 \mathrm{Myr}$ is calculated with a higher metallicity of $Z=0.008$. The bulk of the stars are concentrated into two groups: an apparent main sequence centered on $B-V \sim 0$ mag and an evolved population centered on $B-V \sim 0.8$ mag. The presence of star \#6, which is an SMC supergiant of type K3 I (explained above), provides a practical constraint in choosing the suitable isochrones. Star \#6 is appropriately fitted by two isochrones, those of $18 \mathrm{Myr}$ and $14 \mathrm{Myr}$. The latter is the best fit, but assumes a different metallicity from the other isochrones, i.e. a factor of two higher than the average value for the SMC. This higher metallicity may represent the effect of heavy element enrichment as the K3 star evolves to higher luminosities. These $14 \mathrm{Myr}$ and $18 \mathrm{Myr}$ isochrones also closely reproduce the properties of the main sequence stars, which in their vast majority have an initial mass of roughly $<8 M_{\odot}$.

As far as the exciting star of the $\mathrm{H}_{\text {II }}$ region N33 is concerned, i.e. star \#1, it is indicated by a cross on the color-magnitude diagram. This star is affected by an extinction of $A_{\mathrm{V}}=2.0 \mathrm{mag}$, so it should not belong to the above-mentioned low- and intermediate-mass population, which are mostly spread around $B-V \sim 0$ mag. Star \#1 appears to be a young massive star occurring in a field of old low-mass stars. We also note the color-magnitude diagram position of star \#2, which apparently lies across the $\mathrm{H}$ II region N33. If this star really belongs to the $56 \mathrm{Myr}$ isochrone, as suggested by the diagram, this rules out its association with the $\mathrm{H}$ II region. As for star \#3, it seems to be consistent with the position of a reddened B-type star, hence its association with the $\mathrm{H}_{\text {II }}$ region cannot be excluded at this stage. The second group of stars on the color-magnitude diagram, whose most evolved members gather between $B-V$ colors 0.5 and $1.5 \mathrm{mag}$, have a possible age ranging from $500 \mathrm{Myr}$ to $1 \mathrm{Gyr}$ and a turn-off of $\sim 2 M_{\odot}$. A large number of stars belonging to 


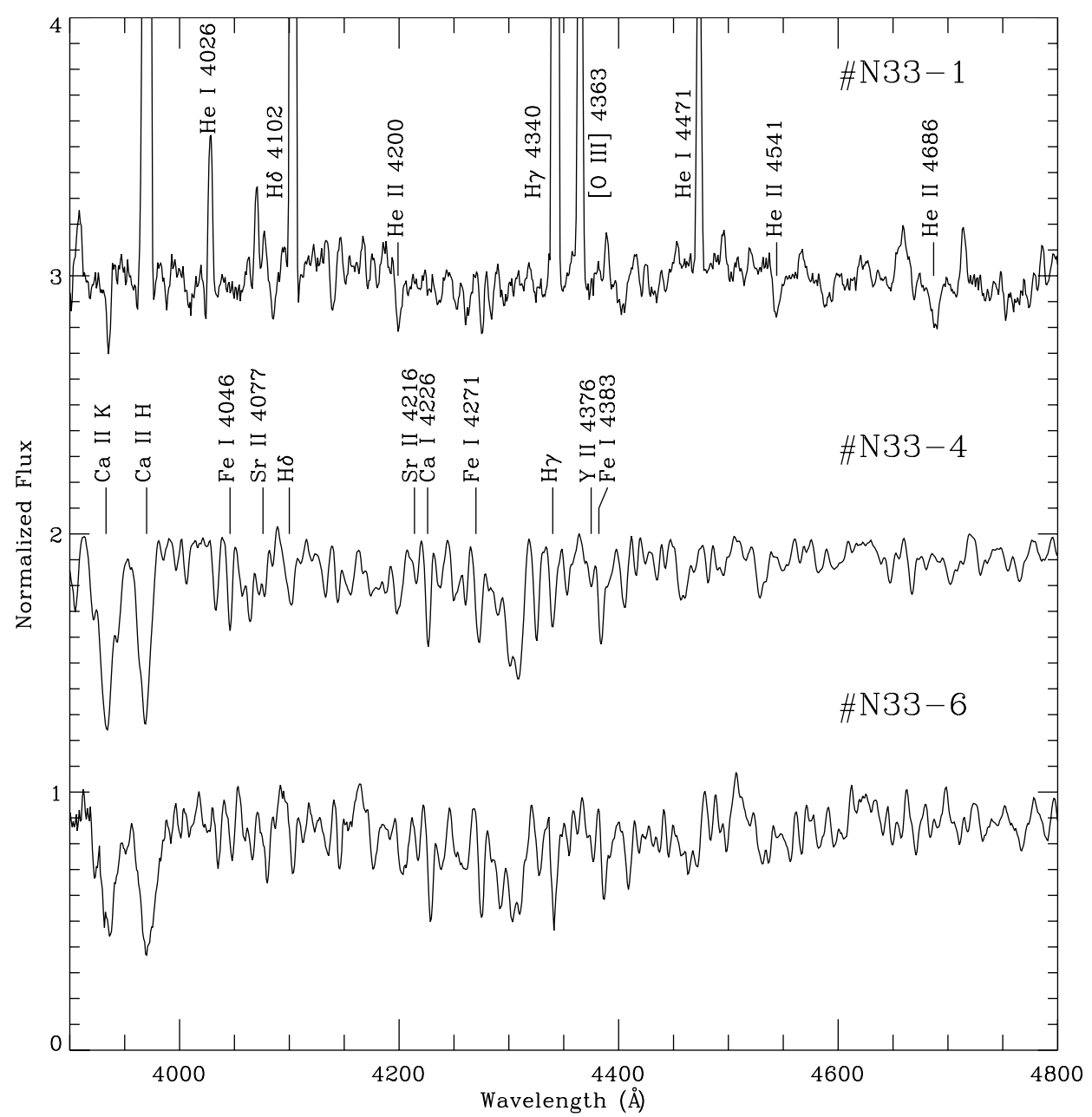

Fig. 6. Spectrograms of three stars observed towards SMC N33. Star \#1 is the exciting source of the compact $\mathrm{H}_{\text {II }}$ region N33. This is a raw spectrum, i.e. it is not corrected for nebular emission lines. Note the He II absorption lines indicating a hot massive star (see Sect. 3.5). Stars \#4 and \#6, lying in the field of N33, are classified as G5 V (Galactic) and K3 I (SMC) respectively.

these older isochrones apparently coincide with the lower part of the $18 \mathrm{Myr}$ and 14 Myr main sequences. We suggest that these various stellar populations may not be locally related to star \#1.

The reason is that the SMC is known to have a complex structure with considerable line-of-sight depth, as shown by several works; we refer to Westerlund (1990) for a review, as well as McGee \& Newton (1981, 1982), Staveley-Smith et al. (1997), and Hatzidimitriou et al. (2005). In particular, Mathewson et al. (1986, 1988) measured the distances of 161 Cepheids in the $\mathrm{SMC}$, using the period-luminosity relation in the infrared. The Cepheids were found to extend from 43 to $75 \mathrm{kpc}$ with a maximum concentration at $59 \mathrm{kpc}$. Since N33 is not part of an OB association (see above Sects. 1 and 4) and more importantly it is an isolated $\mathrm{H}_{\text {II }}$ region, the various stellar populations present in the color-magnitude diagram should not be physically related to it. They are probably distributed along the line of sight over $32 \mathrm{kpc}$. We suggest that the H II region N33 may not be physically associated with this main sequence.

\subsection{Chemical abundances}

An example of the nebular spectra of N33 is shown in Fig. 8, whereas Table 3 lists the corresponding main lines. The spectrum represents an $\mathrm{H}_{\text {II }}$ region, with no He II lines. The two faint lines redward of He I $\lambda 5876$ are $\lambda 5888$ and $\lambda 5890$ in emission.
Both exist in the Orion $\mathrm{H}_{\mathrm{II}}$ region, the first one is unidentified and the second is a [C II] line (Baldwin et al. 2000). Similarly, these two lines are present in the spectrum of the SMC compact $\mathrm{H}$ II region N66A. The ionic abundances with respect to $\mathrm{H}^{+}$ were calculated from nebular lines using the IRAF task ionic of the package NEBULAR (Shaw \& Dufour 1995). The abundance values are listed in Table 4.

To derive the total abundances of a given element, it is necessary to estimate the amount of the element in ionization states not observed in our spectra. We therefore used a set of ionization correction factors (ICFs) to convert into elemental abundances. The absence of the He II line indicates that $\mathrm{He}^{2+} / \mathrm{H}^{+}$is negligible. Moreover, we assume that neutral helium is not important. Thus we assumed that the total $\mathrm{He} / \mathrm{H}$ ratio is just equal to $\mathrm{He}^{+} / \mathrm{H}^{+}$. The total abundance of oxygen was adopted to be the sum of $\mathrm{O}^{+}$ and $\mathrm{O}^{2+}$ abundances. The absence of He II recombination lines in our spectra and the similarity between the ionization potentials of $\mathrm{He}^{+}$and $\mathrm{O}^{++}$implies that the contribution of $\mathrm{O}^{3+}$ is not significant. To obtain the total abundance of nitrogen, we used the usual ICF based on the similarity between the ionization potential of $\mathrm{N}^{+}$and $\mathrm{O}^{+}$(Peimbert \& Costero 1969). The $\mathrm{N}^{+}$abundance does not depend strongly on the electron temperature. The largest errors come from the uncertainty in the $\lambda \lambda 6548$ and 6584 line intensities. Our N result is accurate to within about $30 \%$. As for $\mathrm{Ne}$, the only measurable lines in the optical range are those 


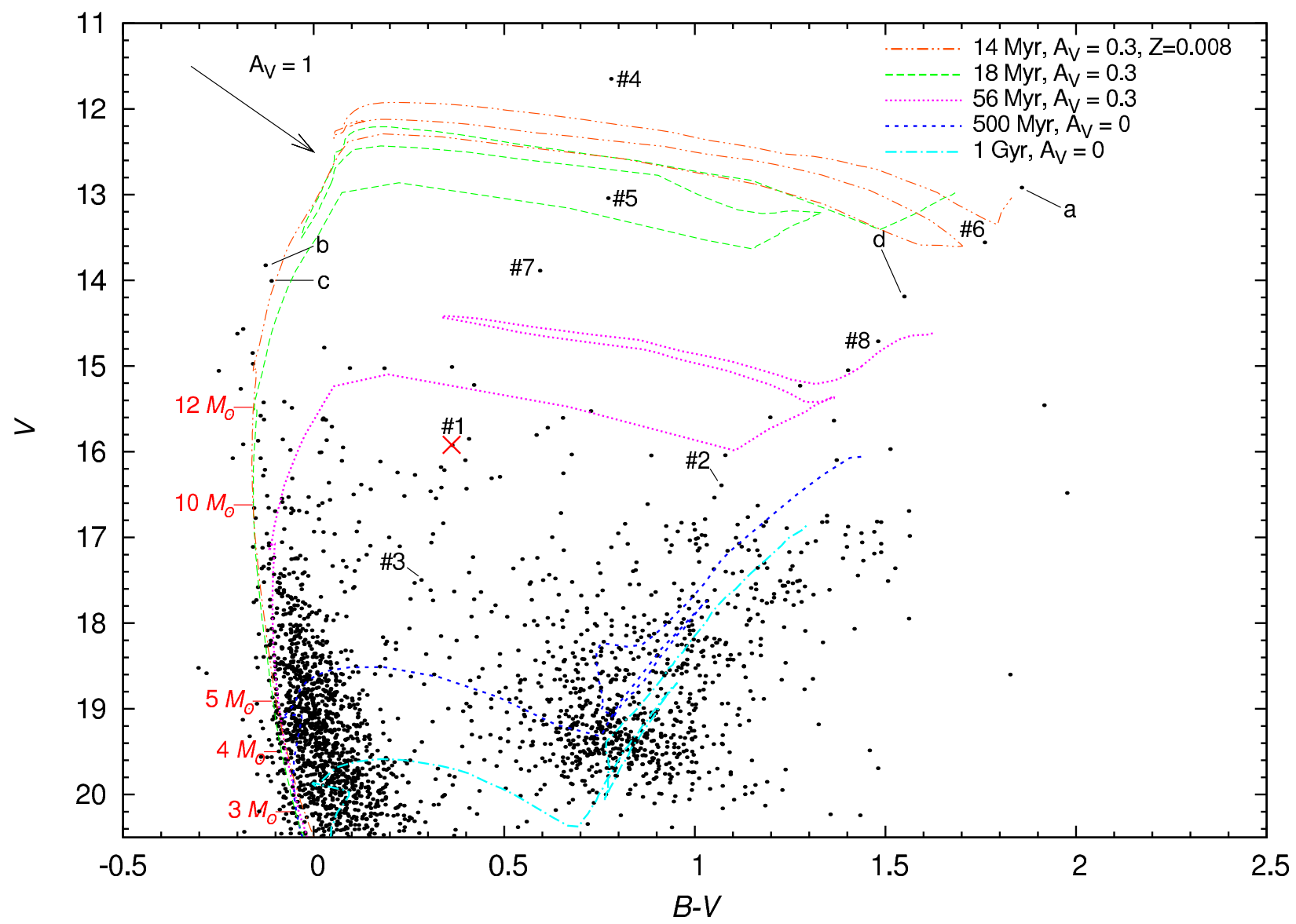

Fig. 7. Color-magnitude, $V$ versus $B-V$, diagram for stars observed toward SMC N33. Five isochrones are shown, $18 \mathrm{Myr}$ with $A_{\mathrm{V}}=0.3$ mag green dashed curve), $56 \mathrm{Myr}$ (violet dotted curve), $500 \mathrm{Myr}$ (blue thick dashed curve), 1 Gyr (aqua dot-dashed curve), computed for a metallicity of $Z=0.004$ (Lejeune \& Schaerer 2001), and 14 My (orange dash-double-dotted curve) for a metallicity of 0.008 and a distance modulus of 18.94 mag. The cross indicates the location of the exciting star of N33. The numbers refer to the stars listed in Table 1 . The letters $a, b, c$, and $d$ belong to the brightest stars of the photometry lying outside the field of Fig. 2. They are located at positions $\alpha / \delta=00: 50: 06.11 /-73: 28: 12.06$, 00:49:51.81/-73:25:16.49, 00:50:03.74/-73:24:16.81, and 00:49:56.82/-73:28:26.17, respectively.

Table 4. Ionic abundances of SMC N33.

\begin{tabular}{lc}
\hline \hline Ion & $\mathrm{N} 33$ \\
\hline $\mathrm{He}^{+} / \mathrm{H}^{+}$ & 0.087 \\
$\mathrm{O}^{+} / \mathrm{H}^{+}\left(\times 10^{5}\right)$ & 3.65 \\
$\mathrm{O}^{++} / \mathrm{H}^{+}\left(\times 10^{5}\right)$ & 7.45 \\
$\mathrm{~N}^{+} / \mathrm{H}^{+}\left(\times 10^{6}\right)$ & 0.92 \\
$\mathrm{Ne}^{++} / \mathrm{H}^{+}\left(\times 10^{6}\right)$ & 8.89 \\
$\mathrm{~S}^{+} / \mathrm{H}^{+}\left(\times 10^{7}\right)$ & 2.10 \\
$\mathrm{Ar}^{++} / \mathrm{H}^{+}\left(\times 10^{7}\right)$ & 4.73 \\
\hline
\end{tabular}

of $\mathrm{Ne}^{2+}$ but the amount of $\mathrm{Ne}^{+}$may be important in the region. We adopted the usual expression of the ionization correction factor of $\mathrm{Ne}$ that assumes that the ionization structure of $\mathrm{Ne}$ is similar to that of O (Peimbert \& Costero 1969).

The total chemical abundances for N33 are presented in Table 5. The most accurately estimated abundances belong to $\mathrm{He}$ and $\mathrm{O}$, which are accurate to within 15 and $20 \%$ respectively. Table 5 also presents the mean abundance values derived for the SMC (Russell \& Dopita 1992). The N33 abundances agree with the SMC mean values. In other words, N33 is, as expected, a low-metallicity ionized nebula.
Table 5. Elemental abundances in SMC N33.

\begin{tabular}{lcc}
\hline \hline Element & N33 & Mean $\mathrm{SMC}^{\dagger}$ \\
\hline $\mathrm{He} / \mathrm{H}$ & 0.087 & 0.081 \\
$\mathrm{O} / \mathrm{H}\left(\times 10^{4}\right)$ & 1.11 & 1.07 \\
$\mathrm{~N} / \mathrm{H}\left(\times 10^{6}\right)$ & 2.76 & 4.27 \\
$\mathrm{Ne} / \mathrm{H}\left(\times 10^{5}\right)$ & 1.34 & 1.86 \\
\hline
\end{tabular}

Notes. ${ }^{(\dagger)}$ Russell \& Dopita (1992).

\subsection{N33 as a HEB}

$\mathrm{N} 33$ should belong to the distinct and rare class of $\mathrm{H}$ II regions in the Magellanic Clouds called HEBs (see Heydari-Malayeri et al. 2010, for a review). In contrast to the typical $\mathrm{H}_{\text {II }}$ regions of the Magellanic Clouds, which are extended structures with sizes of several arc minutes corresponding to physical scales of more than $50 \mathrm{pc}$ and powered by a large number of exciting stars, HEBs are relatively dense and small regions of $\sim 5^{\prime \prime}$ to $10^{\prime \prime}$ in diameter in the optical, corresponding to $\sim 1.5$ to $3.0 \mathrm{pc}$ and excited by a much smaller number of massive stars. Their excitation, as derived from their $[\mathrm{O} \mathrm{III}](\lambda \lambda 4959+5007) / \mathrm{H} \beta$ ratio, is generally larger than that of ordinary Magellanic Cloud $\mathrm{H}$ II regions. For a fixed metallicity, the $\left[\mathrm{O}_{\mathrm{III}}\right] / \mathrm{H} \beta$ ratio increases with the effective 

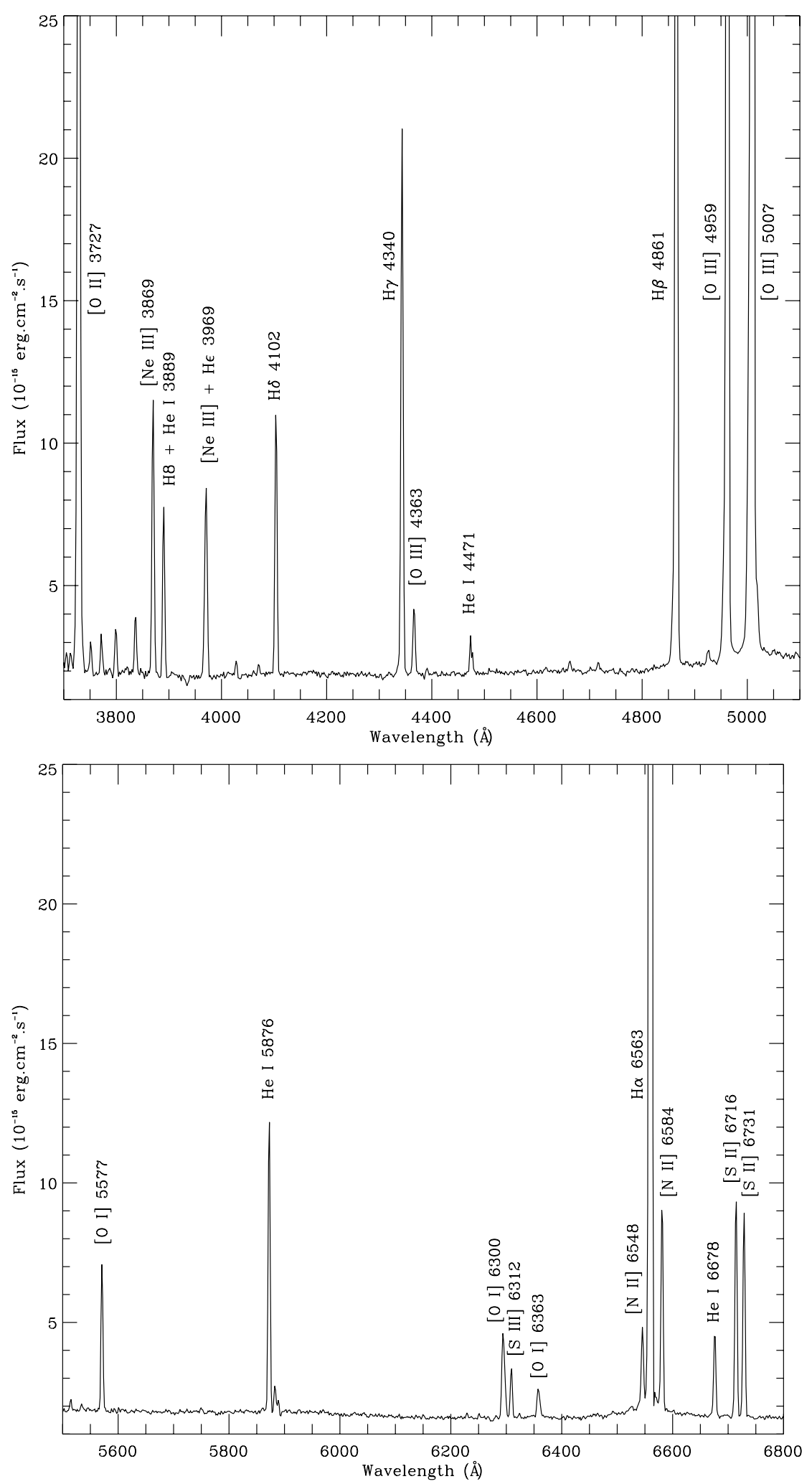

Fig. 8. Typical nebular spectra of the SMC compact $\mathrm{H}$ II region $\mathrm{N} 33$ in the $\mathrm{H} \beta$ (above) and $\mathrm{H} \alpha$ (below) regions.

temperature of the exciting star, as well as with the gas density in homogeneous photoionization models.

N33 qualifies for membership of this category. It is compact, with a mean angular radius of 3 '. 7 , or $1.1 \mathrm{pc}$. The compactness implies a young age, since $\mathrm{H}$ II regions disperse with time and become diffuse. It has a mean $[\mathrm{O} \mathrm{III}] / \mathrm{H} \beta$ ratio of 5.9 , which is higher than the mean value for ordinary extended
H II regions in the Magellanic Clouds (Heydari-Malayeri et al. 2010, and references therein). The electron temperature amounts to $12540 \mathrm{~K}$, which corresponds to excitation by a massive star at least as hot as an O6.5-O7 type at SMC metallicity. The high-excitation auroral transition of doubly ionized oxygen, $\left[\mathrm{O}_{\mathrm{III}}\right] \lambda 4363$, is quite strong, as shown in Figs. 6 and 8. Moreover, the estimated mean extinction over the $\mathrm{H}$ II region, as 


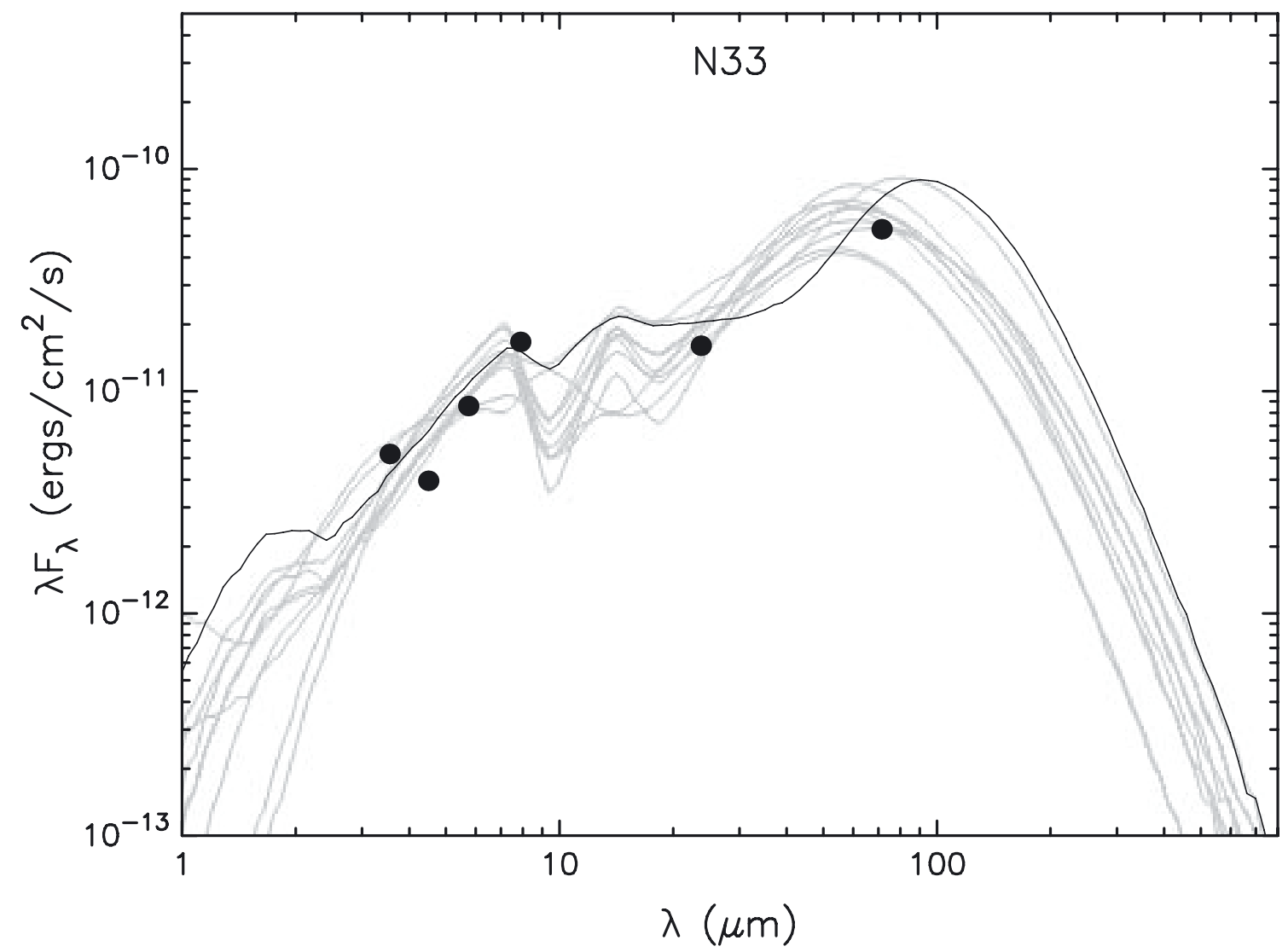

Fig. 9. Mid-IR Spitzer photometry of the H II region N33 fitted using YSO models (Robitaille et al. 2007). The majority of the ten best-fit models suggest the presence of only envelope around the central stellar source of N33. The curve in black represents the best-fit. The derived total luminosity of the nebula according to these models varies between $5 \times 10^{3}$ and $10^{4} L_{\odot}$. One should however be cautious in interpreting these results since N33 is not a YSO; see Sect. 3.8 for details.

derived in the optical, is $A_{\mathrm{V}}=1.5 \mathrm{mag}$. This extinction peaks at 2.5 mag towards the northeast border, where the nebula is probably in contact with a molecular cloud of unknown characteristics. This association with a molecular cloud is another characteristic of HEBs. Moreover, in the plot comparing Magellanic Clouds compact $\mathrm{H}_{\text {II }}$ regions among them, N33 appears to be one of the most excited and brightest members of this class in the SMC (Meynadier \& Heydari-Malayeri 2007).

\subsection{Spitzer SED fitting}

We used our Spitzer photometry to construct the mid-IR SED of N33 and assess the behavior of the circumstellar dust of the nebula. We fitted this SED with the library of YSO models by Robitaille et al. (2006) using the online SED fitting tool, provided by these authors (Robitaille et al. 2007) ${ }^{2}$. Figure 9 shows the ten best-fit models to this SED. Only three of these models include both circumstellar envelopes and disks. The majority of the best-fit YSO models contain only one envelope around the central stellar source of N33 with an outer radius varying between $3 \times 10^{4}$ and $10^{5} \mathrm{AU}$. The derived total luminosity of the nebula according to these models varies between $5 \times 10^{3}$ to $10^{4} L_{\odot}$. This agrees with the luminosity of $\sim 5 \times 10^{4} L_{\odot}$, derived from IRAS fluxes considering that the IRAS SED peaks at $\sim 60 \mu \mathrm{m}$. Unfortunately, the blue part of the complete SED of N33, which includes the stellar source, could not be properly

\footnotetext{
2 And available at

http://caravan.astro.wisc.edu/protostars
}

fitted. There are four models available in the library of Robitaille et al. (2006) that include stellar sources with $T_{\text {eff }}$, corresponding to the spectral type of the central star of N33 and a mass of about $25 M_{\odot}$. However, their fit to the blue part of our SED was not successful because there were few available measurements in the optical. In all cases, although SED fitting is an interesting exercise, its results should not be over-interpreted for the simple reason that $\mathrm{N} 33$ is not a YSO.

\section{Discussion}

$\mathrm{N} 33$ is quite noteworthy in that it is a isolated $\mathrm{H}_{\text {II }}$ region in this area of the SMC. In contrast to typical $\mathrm{H}_{\text {II }}$ regions in the Magellanic Clouds, which have neighboring $\mathrm{H}_{\text {II }}$ regions, N33 differs from any typical emission nebula. Thus it constitutes an interesting case for studies regarding the physical conditions in which massive stars form in isolation. Observational findings suggest that massive star formation is a collective process, i.e. that massive stars form in cluster environments (e.g., Zinnecker \& Yorke 2007; Schilbach \& Röser 2008; Gvaramadze \& Bomans 2008) and that the mass of the most massive star in a cluster is correlated with the mass of the cluster itself (Elmegreen 2000; Weidner \& Kroupa 2006). Nevertheless, other observational results imply that massive stars do not necessarily form in clusters but can be formed either as isolated stars or in very small groups. According to a statistical study by de Wit et al. (2005), nearly $95 \%$ of Galactic O population is located in clusters or $\mathrm{OB}$ associations. This means that a small percentage, about $5 \%$, of high mass stars may form in isolation, in very good 
agreement with the finding by Parker \& Goodwin (2007). Even in the Galactic center region, a number of Wolf-Rayet stars and O supergiants might have formed in isolation (Mauerhan et al. 2010). Lamb et al. (2010) observed eight apparently isolated O stars in the SMC. Among the six non-runaway cases, there is no evidence of clustering in three of them, which remain interesting candidates for isolated massive-star formation. By isolation, we mean not clearly originating in an $\mathrm{OB}$ association. This definition therefore excludes runaway massive stars, which are thought to result from either dynamical interaction in massive dense clusters, or via a kick from a supernova explosion in a binary system (e.g., Gvaramadze et al. 2009; Pflamm-Altenburg \& Kroupa 2010, and references therein). Parker \& Goodwin (2007) give a different definition for an isolated massive star: an Otype star belonging to a cluster whose total mass is $<100 M_{\odot}$ and moreover is devoid of B-type stars $\left(10<M / M_{\odot}<17.5\right)$. In the LMC, several massive runaway candidates have been reported (e.g. Heydari-Malayeri et al. 2000; Evans et al. 2010; Gvaramadze et al. 2010). Similarly, in the SMC a dozen OB runaway candidates were reported via detection of their bow shocks using Spitzer data (Gvaramadze et al. 2011).

In any case, the massive star(s) powering N33 cannot be runaway(s) for three main reasons: 1) N33 is not linked to any OB association; 2) it seems impossible that a massive star carries its $\mathrm{H}_{\text {II }}$ region during the ejection, although ejection into a molecular cloud cannot be a priori excluded; and 3) were N33 to contain more than one (probably exciting) stars, this situation would make the ejection hypothesis still less probable. On the other hand, the possibility that a massive star ejected into a molecular cloud creates an $\mathrm{H}$ II region around itself has never been encountered.

To obtain a clear picture of how scattered the cold interstellar material is in the general area of N33, we investigate the distribution of molecular clouds and known young stellar objects (YSOs) in the vicinity $(\sim 200 \mathrm{pc})$ of N33. CO surveys of the SMC reveal a concentration of molecular clouds in the southern end of this galaxy (Fukui \& Kawamura 2010; Mizuno et al. 2001; Stanimirović et al. 2000, 1999; Blitz et al. 2007; Bolatto et al. 2007; Leroy et al. 2007; Bot et al. 2010). However, the available $\mathrm{CO}$ observations do not detect the $\mathrm{N} 33 \mathrm{H}$ II region, maybe because of a lack of sensitivity or resolution or for other reasons. The nearest molecular cloud to N33 is centered at a projected distance of $\sim 230$ pc (Fukui \& Kawamura 2010, and references therein). As for YSOs, we used the catalog of candidates for these objects derived with Spitzer from the $S^{3} \mathrm{MC}$ survey (Bolatto et al. 2007). In this area, we found five candidate YSOs, three of them being quite remote from N33 at distances of between about 48 and $80 \mathrm{pc}$, hence we do not consider them to be related to N33. On the other hand, we note that the two remaining candidate YSOs, named S3MC_J004930.12-732623.42 and S3MC_J004929.07-732658.56 (see Bolatto et al. 2007, Table 4), are projected much closer to N33 at distances of about 5 and 7 pc, respectively (Fig. 3). The nature of these red sources as true YSOs still remains to be verified, and the possibility of a connection between N33 and these objects certainly requires further investigation, preferably with mid- and near-IR spectroscopy of these sources. We also note that the $S^{3} \mathrm{MC}$ team did not identify $\mathrm{N} 33$ as a candidate YSO.

It has been suggested that isolated field OB stars originate in a different mode of star formation from their counterparts in associations. Massey (2002) and Kroupa \& Weidner (2003) make the case that the field star initial mass function (IMF) might differ from that in $\mathrm{OB}$ associations/clusters. More explicitly, Galactic-field IMFs for early-type stars cannot, under any circumstances, be a Salpeter power-law, i.e. $\alpha=2.35$, but must have a steeper exponent, $\alpha \geq 2.8$ (Kroupa \& Weidner 2003) Similarly, special physical conditions have been called up to account for the formation of very high-mass isolated stars in the bulge of the interacting Whirlpool galaxy, M51 (Lamers et al. 2002). According to these authors, the formation of the bulge stars in M 51 seems to be in line with theoretical predictions that isolated massive-star formation might take place in clouds where $\mathrm{H}_{2}$, [O I $63 \mu \mathrm{m}$, and [C $\left.\mathrm{II}\right] 158 \mu \mathrm{m}$ are the dominant coolants (Norman \& Spaans 1997; Mihos et al. 1999). These conditions are expected to occur in regions of low CO and dust contents because of the low metallicity, where the optical depth is $A_{\mathrm{V}} \leq 1 \mathrm{mag}$ and the presence of a hot source can dissociate the CO molecules (Lamers et al. 2002). The isolated massive stars might therefore be the result of these physical conditions and the luminous nucleus (situated at a distance between 90 and $270 \mathrm{pc}$ ). For comparison, the metallicity of SMC N33 is about a factor of 10 lower than solar. However, no major COdissociating hot source is present. The pre-eminent massive star cluster N66/NGC 346 (Heydari-Malayeri \& Selier 2010, and references therein) lies some $1400 \mathrm{pc}$ away. Could this source have a similar effect as the M51 nucleus? Another similarity with M 51 is that the SMC is also an interacting galaxy.

We note that in contrast to the above-mentioned proposal for the two different formation modes for cluster and isolated stars, Oey et al. (2004) find that the formation of field massive stars is a continuous process in associations. More specifically, the field stars do not originate from any different star-formation mode. This conclusion is based on an empirical census of uniformlyselected massive-star candidates distributed all-over the SMC in more than 100 star clusters containing at least 3 to about 80 stars. Moreover, according to their Monte Carlo simulations, single, field $\mathrm{OB}$ stars are usually the most massive member of a group of smaller stars. This result is consistent with the idea that there is both a universal IMF and universal $N_{*}^{-2}$ clustering law, which extends to $N_{*}=1$. Jointly, these laws imply that the fraction of field OB stars typically ranges from about $35 \%$ to $7 \%$ for most astrophysical situations.

Kauffmann \& Pillai (2010) suggest that massive stars might form only in molecular clouds possessing a minimum mass depending on the cloud size: $M(r)>870 M_{\odot}(r / \mathrm{pc})^{1.33}$. Thus, massive-star formation requires a large mass being concentrated in a relatively small volume. Clouds below this threshold might still form stars and clusters of up to intermediate mass (such as the Galactic Taurus, Ophiuchus, and Perseus molecular clouds). One can infer from this finding that molecular clouds with a mass slightly above this limit can form one or a couple of massive stars. N33 may represent such a case, but the problem is not at all solved, since it is then unclear why in this area of the SMC only one cloud happens to be massive enough to give rise to massive stars. In other words, we have yet to ascertain which physical conditions inhibit the aggregation of smaller clumps into massive clouds.

In any case, the isolated massive star(s) powering N33 cannot be a result of the competitive accretion process (Bonnell et al. 1997, 2001). According to this scenario, developing protostars in their natal molecular clouds compete with each other to gather mass. The protostars accrete mass with a rate that depends on their location within the protocluster. They use the same reservoir of gas to grow. Hence, the protostars nearest the center, where the potential well is deep and gas densities are higher, have the highest accretion rates. This model is synonymous with collective massive-star formation. 
There is no doubt that star \#1, the exciting source of the $\mathrm{H}$ II region, is a massive star (of derived spectral type O6.5$\mathrm{O} 7 \mathrm{~V}$ ). We also note that this star constitutes the main object of the isolated massive-star formation debate presented in this paper. In contrast, the nature of the two neighboring stars \#2 ( $V=$ $16.39, B-V=1.07 \mathrm{mag})$ and $\# 3(V=17.50, B-V=0.28 \mathrm{mag})$ is unclear. The presence of these stars raises several questions: Are they massive? Are they physically related to the compact H II region and in particular to the massive star \#1? The present study cannot provide firm responses to these questions. We have found that if there is a clustering in the region this occurs at a scale of about $3 \mathrm{pc}$ and smaller, which actually covers the $1 \mathrm{pc}$ separation between stars \#1, \#2, and \#3. We note, however, that the color of star \#2 ( $B-V=1.07 \mathrm{mag})$ does not match that expected for a massive star assuming that stars \#1 and \#2 are more or less equally affected by reddening. As for star \#3, its absolute magnitude and color are compatible with a B-type star of mass about $15 M_{\odot}$. Further high-resolution observations, both imaging and spectroscopy, are necessary to probe the exact nature of this star and its relation to the $\mathrm{H}$ II region.

HEBs are usually located adjacent to ordinary giant $\mathrm{H}_{\text {II }}$ regions or seen lying across them. This implies that their formation is the consequence of triggering by a previous generation of massive stars in the complex (Elmegreen \& Lada 1977; Whitworth et al. 1994; Deharveng et al. 2009, and references therein). However, this is not the case for N33 whose massive star(s) has(have) formed in isolation. Another example of an SMC HEB that has formed in isolation is N81, which was studied using HST imaging and spectroscopy (Heydari-Malayeri et al. 1999a, 2002; Martins et al. 2004). It seems, however, that N33 is a comparatively more attractive candidate for isolated massivestar formation. N81 is an isolated H II region in Shapley's wing. Nevertheless, it is certainly powered by at least two main $\mathrm{O}$ type stars, which are apparently part of a small cluster of massive stars detected with the HST spatial resolution (Heydari-Malayeri et al. 1999a). The massive star at the origin of N33 has most certainly formed in an isolated, small molecular cloud, the mass and physical characteristics of which are not known.

It will be interesting to derive the IMF for such small massive star clusters formed in isolation, and compare it with that of massive stars formed in OB associations. We emphasize that individual studies of a variety of cases is essential in parallel with global, statistical investigations. These small clusters provide relatively simpler situations for studying the IMF because they involve a smaller number of physical factors because of their isolation. In the case of N33, it seems that the initial mass of the exciting star is $\sim 40 M_{\odot}$. It is unsurprising that the highest masses in small clusters be lower than those in OB associations. Indeed small clusters originate in molecular clouds with smaller masses. To advance the study of the mass distribution in small clusters, high resolution techniques in the optical and IR, both imaging and spectroscopy, environmental as well as stellar, are required.

\section{Concluding remarks}

This paper has presented the first detailed study of SMC N33 using imaging and spectroscopy in the optical obtained at the ESO NTT, as well as from the Spitzer and 2MASS data archives. We have derived a number of physical characteristics of N33 and ascertained its powering source. This compact $\mathrm{H}$ II region of $\sim 7$ '. $4(2.2 \mathrm{pc})$ in diameter belongs to a small class of HEBs in the MCs. In contrast to other members of this class, N33 is not associated with any OB association. This object is excited by a massive star of type $06.5-\mathrm{O} 7 \mathrm{~V}$, and represents an interesting case of isolated massive-star formation in the SMC.

Acknowledgements. We would like to thank the people in charge of Spitzer data archives, and particularly the PIs of the $S^{3} \mathrm{MC}$ and SAGE-SMC projects, Dr. Alberto D. Bolatto, University of Maryland, and Dr. Karl D. Gordon, University of Arizona, respectively. Our special thanks go also to the 2MASS facility for the data used in this study. D.A.G. kindly acknowledges financial support from the German Aerospace Center (DLR) through grant 50 OR 0908. We are indebted to Prof. Vassilis Charmandaris, Crete University, for his advice and help in using the Spitzer data. We would like also to thank Dr. Nolan R. Walborn, Space Telescope Institute, Baltimore, and Dr. Fabrice Martins, GRAAL, Montpellier, for commenting on a preliminary version of this paper. Last but not least, we are grateful to an anonymous referee for his meticulous review of the manuscript and several important suggestions that led to a much improved presentation of this research.

\section{References}

Baldwin, J. A., Verner, E. M., Verner, D. A., et al. 2000, ApJS, 129, 229 Battinelli, P. 1991, A\&A, 244, 69

Bica, E. L. D., \& Schmitt, H. R. 1995, ApJS, 101, 41

Blitz, L., Fukui, Y., Kawamura, A., et al. 2007, in Protostars and Planets V, ed. B. Reipurth et al. (Tuscon: Univ. of Arizona Press), 81

Bolatto, A. D., Simon, J. D., Stanimirović, S., et al. 2007, ApJ, 655, 212

Bonnell, I. A., Bate, M. R., Clarke, C. J., \& Pringle, J. E. 1997, MNRAS, 285, 201

Bonnell, I. A., Bate, M. R., Clarke, C. J., \& Pringle, J. E. 2001, MNRAS, 323, 785

Bot, C., Rubio, M., \& Boulanger, F., et al. 2010, A\&A, 524, A52

Bouchet, P., Lequeux, J., Maurice, E., et al. 1985, A\&A, 149, 330

Castilho, B. V., Gregorio-Hetem, J., Spite, F., et al. 2000, A\&A, 364, 674

Charmandaris, V., Heydari-Malayeri, M., \& Chatzopoulos, E. 2008, A\&A, 396, 255

Cignoni, M., Sabbi, E., Nota, A., et al. 2009, AJ, 137, 3668

Davies, R. D., Elliott, K. H., \& Meaburn, J. 1976, MNRAS, 81, 89 (DEM)

Deharveng, L., Zavagno, A., Schuller, F., et al. 2009, A\&A, 496, 177

Dekker, H., Delabre, B., \& Dodorico, S. 1986, SPIE, 627, 339D

de Wit, W. J., Testi, L., Palla, F., \& Zinnecker, H. 2005, A\&A, 437, 247

D'Odorico, S., Beletic, J. W., Amico, P., et al. 1998, SPIE, 3355, 507

Elmegreen, B. G. 2000, ApJ, 539, 342

Elmegreen, B. G., \& Lada, C. J. 1977, ApJ, 214, 725

Evans, C. J., Walborn, N. R., Crowther, P. A., et al. 2010, ApJ, 715, L74

Filipović, M. D., Haynes, R. F., White, G. L., \& Jones, P. A. 1998, A\&AS, 130, 421

Fukui, Y., \& kawamura, A. 2010, ARA\&A, 48, 547

Gomez, M., Hartmann, L., Kenyon, S. J., \& Hewett, R. 1993, AJ, 105, 1927

Gouliermis, D., Kontizas, M., Korakitis, R., et al. 2000, AJ, 119, 1737

Gray, R. O., \& Corbally, C. J. 2009, Stellar Spectral Classification (Princeton Univ. Press)

Gvaramadze, V. V., \& Bomans, D. J. 2008, A\&A, 490, 1071

Gvaramadze, V. V., Gualandris, A., \& Portegies Zwart, S. 2009, MNRAS, 396, 570

Gvaramadze, V. V., Kroupa, P., \& Pflamm-Altenburg, J. 2010, A\&A, 519, A33 Gvaramadze, V. V., Pflamm-Altenburg, J., \& Kroupa, P. 2011, A\&A, 525, A17 Hatzidimitriou, D., Stanimirović, S., Maragoudaki, F., et al. 2005, MNRAS, 360, 1171

Helou, G., \& Walker, D. W. 1988, Infrared Astronomical Satellite (IRAS) Catalogs and Atlases, The Small Scale Structure Catalog, NASA RP-1190 (Washington: GPO), 7

Henize, K. G. 1956, ApJS, 2, 315

Henize, K. G., \& Westerlund, B. E. 1963, ApJ, 137, 747

Heydari-Malayeri, M., \& Selier, R. 2010, A\&A, 517, A39

Heydari-Malayeri, M., Rosa, M. R., Zinnecker, H., Deharveng, L., \& Charmandaris, V. 1999a, A\&A, 344, 848

Heydari-Malayeri, M., Charmandaris, V., Deharveng, L., Rosa, M. R., \& Zinnecker, H. 1999b, A\&A, 347, 841

Heydari-Malayeri, M., Royer, P., Rauw, G., \& Walborn, N. R. 2000, A\&A, 361, 877

Heydari-Malayeri, M., Rosa, M. R., Schaerer, D., Martins, F., \& Charmandaris, V. 2002, A\&A, 381, 951

Heydari-Malayeri, M., Rosa, M. R., Charmandaris, V., et al. 2010, The Impact of HST on European Astronomy, ed. F. D. Macchetto, Astrophysics and Space Science Proc., 31

Hodge, P. 1985, PASP, 97, 530

Howarth, I. D. 1983, MNRAS, 203, 301

Indebetouw, R., Johnson, K. E., \& Conti, P. 2004, AJ, 128, 2206 
Jaschek, C., \& Jaschek, M. 1987, The classification of stars (Cambridge Univ. Press)

Jacoby, G. H., \& De Marco, O. 2002, AJ, 123, 269

Johnson, M. D., Levitt, J. S., Henry, R. B. C., et al. 2006, Planetary Nebulae in Our Galaxy and Beyond, ed. M. J. Barlow, \& R. H. Méndez, Proc. IAU Symp., 234 [arXiv: astro-ph/0605099]

Kauffmann, J., \& Pillai, T. 2010, ApJ, 723, L7

Koornneef, J. 1983, A\&A, 128, 84

Kroupa, P., \& Weidner, C. 2003, ApJ, 598, 1076

Kwitter, K. B., \& Henry, R. B. C. 2001, ApJ, 562, 804

Lindsay, E. M. 1961, AJ, 66, 169

Lamb, J. B., Oey, M. S., Werk, J. K., \& Ingleby, L. D. 2010, ApJ, 725, 1886

Lamers, H. J. G. L. M. 2005, Proc. IAU Symp., ed. Cesaroni et al. (Cambridge Univ. Press), 227, 303

Lamers, H. J. G. L. M., Panagia, N., Scuderi, S., et al. 2002, ApJ, 566, 818 Landolt, A. U. 1992, AJ, 104, 340

Laney, C. D., \& Stobie, R. S. 1994, MNRAS, 266, 441

Larson, R. B. 1995, MNRAS, 272, 213

Lee, M.-Y., Stanimirović, S., Jürgen, O., et al. 2009, AJ, 138, 1101

Lejeune, T., \& Schaerer, D. 2001, A\&A, 366, 538

Leroy, A., Bolatto, A., Stanimirović, S., et al. 2007, ApJ, 658, 1027

Levesque, E. M., Massey, P., Olsen, K. A. G., et al. 2006, ApJ, 645, 1102

Martins, F., \& Plez, B. 2006, A\&A, 457, 637

Martins, F., Schaerer, D., Hillier, D. J., \& Heydari-Malayeri, M. 2004, A\&A, 420, 1087

Martins, F., Schaerer, D., \& Hillier, D. J. 2005, A\&A, 436, 1049

Massey, P. 2002, ApJS, 141, 81

Mathewson, D. S., Ford, V. L., \& Visvanathan, N. 1986, ApJ, 301, 664

Mathewson, D. S., Ford, V. L., \& Visvanathan, N. 1988, ApJ, 333, 617

Mauerhan, J., Cotera, A., Dong, H., et al. 2010, ApJ, 725, 188
McGee, R. X. \& Newton, L. M. 1981, PASAu, 4, 189 McGee, R. X., \& Newton, L. M. 1982, PASAu, 4, 308 Meyssonnier, N., \& Azzopardi, M. 1993, A\&AS, 102, 451

Meynadier, F., \& Heydari-Malayeri, M. 2007, A\&A, 461, 565 Mihos, J. C., Spaans, M., \& McGaugh, S. S. 1999, ApJ, 515, 89 Mizuno, N., Rubio, M., Mizuno, A., et al. 2001, PASJ, 53, L45 Norman, C. A., \& Spaans, M. 1997, ApJ, 480, 145

Oey, M. S., King, N. L., \& Parker, J. Wm. 2004, AJ, 127, 1632

Parker, R. J., \& Goodwin, S. P. 2007, MNRAS, 380, 1271

Peimbert, M., \& Costero, R. 1969, Bol. Obs. Tonantzintla y Tacubaya, 5, 3

Pflamm-Altenburg, J., \& Kroupa, P. 2010, MNRAS, 404, 1564

Prévot, M. L., Lequeux, J., Prevot, L., et al. 1984, A\&A, 132, 389

Robitaille, T. P., Whitney, B. A., Indebetouw, R., et al. 2006, ApJS, 167, 256

Robitaille, T. P., Whitney, B. A., Indebetouw, R., \& Wood, K. 2007, ApJS, 169, 328

Russell, S. C., \& Dopita, M. A. 1992, ApJ, 384, 508

Schilbach, E., \& Röser, S. 2008, A\&A, 489, 105

Shapley, H. 1940, BHarO, 914, 8

Shaw, R. A., \& Dufour, R. J. 1995, PASP, 107, 896

Stanimirović, S., Staveley-Smith, L., Dickey, J. M., et al. 1999, MNRAS, 302, 417

Stanimirović, S., Staveley-Smith, L., van der Hulst, J. M., et al. 2000, MNRAS, 315,791

Staveley-Smith, L., Sault, R. J., Hatzidimitriou, et al. 1997, MNRAS, 289, 225

Weidner, C., \& Kroupa P. 2006, MNRAS, 365, 1333

Westerlund, B. E. 1990, A\&ARv, 2, 29

Whitworth, A. P., Bhattal, A. S., Chapman, S. J., et al. 1994, MNRAS, 268, 291

Wilke, K., Stickel, M., Haas, M., et al. 2003, A\&A, 401, 873

Zinnecker, H., \& Yorke, H. W. 2007, ARA\&A, 45, 481 